Angel Guillén Zanón

Inspector Coordinador de la IGSAP

Cuerpo Superior de ACE

\title{
La Técnica del Grupo Nominal (*)
}

SUMARIO: I. INTRODUCCION. II. EL ANALISIS DE PROBLEMAS Y LA TOMA DE DECISIONES. III. LA TECNICA DEL GRUPO NOMINAL COMO INSTRUMENTO PARA EL ANALISIS Y LA TOMA DE DECISIONES. 1. El grupo nominal. 2. Las reuniones convencionales. 3. La TGN como alternativa. IV. OBJETIVOS DE LA TGN. V. CARACTERISTICAS DE LA TGN. VI. DESARROLLO Y FASES DE UNA TGN. 1. Factores que intervienen en la TGN. 1.1. El Director de la TGN. 1.2. Participantes. 1.3. Preguntas a formular. 1.4. Votaciones. 2. Tareas preparatorias. 2.1. La sala de reunión. 2.2. El material. 2.3. Explicaciones de objetivos. 3. El desarrollo del proceso. 3.1. Generación silenciosa de ideas por escrito (Fase 1."). 3.2. Registro de ideas (Fase 2.") . 3.3. Debate aclaratorio (Fase 3." ) 3.4. Votación para la priorización de las ideas (Fase 4." ) 3.5. Discusión de la votación preliminar (Fase 5.p) 3.6. Segunda votación o votación definitiva (Fase 6."). VII. VENTAJAS DE LA TGN. VIII. INCONVENIENTES DE LA TGN. IX. FACTORES DE EXITO DE UNA TGN. X. IMPLANTACION EN EL SECTOR PUBLICO. XI. EXAMEN DE UN CASO PRACTICO. 1. Antecedentes. 2. Objetivo. 3. Selección de participantes. 4. Cuestiones formuladas. 5. Resultados obtenidos. 5.1. Evaluación y comentarios a la TGN del grupo primero. 5.2. Evaluación y comentarios a la TGN del grupo segundo. 5.3. Examen comparado de los resultados obtenidos. 6. Conclusiones.

\section{INTRODUCCION}

Angelo L. Fortuna, Director de Desarrollo de Recursos Humanos y Organización de ARA Services, afirma que «los alquimistas no lograron, después de una larga búsqueda, convertir los metales de base en metales nobles y, sin embargo, los creadores de la Técnica del Grupo Nominal (TGN) han logrado, en mucho menos tiempo, algo probable-

(*) En la búsqueda de documentación para la elaboración de este trabajo ha intervenido doña Isabel Pastor Garcia-Moreno, del Cuerpo Superior de Administradores Civiles del Estado, Directora de Programa de la IGSAP.

Documentación Administrativa / n.' 223 (julio-septiembre 1990) 
mente más fructífero para las organizaciones: convertir las pesadas sesiones de grupo en oportunidades de oro para la resolución de problemas» (1). Se trata de combatir lo que el citado autor denomina «la crisis energética del Management», a saber: que el directivo standard emplea alrededor de un 80 por 100 de su tiempo de un día de trabajo en reuniones con comités. Pero, lo que es peor, las reuniones jamás concluyen. De ellas, en pocas ocasiones se extraen conclusiones aptas para ser utilizadas directamente en la toma de decisiones y, por ello, los directivos se ven envueltos en un círculo vicioso que es causa directa e inmediata del síndrome conocido como «una reunión conduce inevitablemente a otra reunión».

Hoy en día, los estudiosos de la Teoría de la Organización son conscientes de este dato, $y$, en consecuencia, quieren terminar con este proceso sin fin, para lo que existe una continua buisqueda de mecanismos que potencien la eficacia de las reuniones.

Efectivamente, es posible explicar el anterior fenómeno en breves palabras: las reuniones tradicionales, en su orden del día suelen contener un último apartado denominado «Conclusiones», pero, a la hora de convertirlo en realidad, el encargado de levantar acta tenderá a hacer un breve resumen de lo hablado, a señalar la importancia de alguna cuestión en particular y, finalmente, recogerá la sugerencia de algún participante, quizá del presidente, de que resulta imprescindible convocar una futura reunión donde se discuta en profundidad el asunto de que se trate. ¿Cuál habrá sido el resultado de la reunión? Está claro: la convocatoria de otra reunión. ¿El objetivo de la reunión?: otra reunión, y así hasta... ¿dónde?

La TGN es, en realidad, una técnica creativa para el análisis de problemas con un propósito especial: combinar opiniones individuales para permitir la toma de decisiones. Es una técnica que permite incrementar la capacidad creativa del grupo para generar ideas, descomponer problemas y proponer soluciones. Ello significa que merced a ella los participantes agregan opiniones individuales y llegan a elaborar opiniones de grupo.

La TGN tiene sus orígenes en el pensamiento de André DELBECQ y ANDREW VAN DE VEN sobre las reuniones de grupo, citándose como fecha de despegue 1968. Esta técnica ha sido utilizada en el sector privado, pasando posteriormente al sector público de una forma muy limitada, utilizándose sólo en aquellos campos que implicaban planificación de servicios sociales como la educación o la sanidad.

(1) Esto lo explica Angelo L. Fortuna, Director de Desarrollo de Recursos Humanos y Organización de ARA Services. Inc., en su introducción a la obra de DELBECQ, VAN DE VEN y GuSTAFson, Group Tecbniques for Program Planning. A Guide to Nominal Group and Delphi Processes, Ed. Green Briar Press, p. 13. 


\section{EL ANALISIS DE PROBLEMAS Y LA TOMA DE DECISIONES}

Las organizaciones modernas se caracterizan entre otras cosas por la necesidad de establecer estrategias a medio y largo plazo, así como por la urgencia de abordar los problemas concretos mediante una toma de decisiones eficaz.

Para efectuar esta apremiante tarea se vienen utilizando desde mediados de la década de los cincuenta un conjunto de técnicas analíticas y creativas, como instrumentos idóneos para alumbrar soluciones y alternativas, si bien unas y otras difieren por:

- el método;

- el tipo de problemas que pueden abordar;

- la utilización, o no, del grupo como instrumento de apoyo.

Las técnicas analíticas utilizan un método deductivo, abordan problemas fundamentalmente cerrados, de límites claros y delimitados y se apoyan en la reflexión individual y en un proceso lógico y sistemático que es preciso recorrer necesariamente hasta conseguir el diagnóstico de la situación planteada.

Las técnicas creativas se basan fundamentalmente en la intuición y creatividad, se dirigen especialmente a la solución de problemas abiertos (de límites imprecisos) y se apoyan para su desarrollo en el grupo, aun cuando se generen de forma individual.

Las técnicas creativas permitirán abordar con mayor libertad la producción de ideas y soluciones alternativas, así como buscar las causas y motivos que pueden incidir en una situación de hecho, ahorrándose el largo proceso deductivo propio de las técnicas analíticas.

Mientras que entre estas últimas se puede citar, entre otras, el método Kepner-Tregoe, el Análisis morfológico y el Listado de control, etc., entre las primeras es preciso citar el Brainstorming, el Braiwrinting, el método Delphi y la TGN.

Lo que llama la atención es el amplio impacto que la TGN ha logrado en muy poco tiempo en la práctica totalidad de las organizaciones privadas y la lentitud de su incorporación al sector público, en donde no se han asumido aún todas las posibilidades de su aplicación.

En el caso concreto de la TGN los puntos de partida de DELBECQ y VAN DE VEN fueron las teorías sociopsicológicas sobre reuniones para la toma de decisiones, los planteamientos de la Ciencia de la Administración sobre las opiniones de los grupos y los estudios sociológicos sobre la participación de los ciudadanos en la planificación. Desde entonces la TGN ha pasado a tener un amplio reconocimiento y ha empezado a ser aplicada en el sector público. 


\section{LA TECNICA DEL GRUPO NOMINAL COMO INSTRUMENTO PARA EL ANALISIS Y LA TOMA DE DECISIONES}

La TGN es, esencialmente, una reunión de grupo, si bien con unas características especiales. El grupo se utiliza como punto de apoyo para generar ideas, sin que su producción se deba a un proceso interactivo, sino a una generación silenciosa e individual de ideas. Es decir, se trata de una técnica que funciona en grupo en un aspecto puramente nominal o formal. Es, fundamentalmente, su capacidad para lograr ciertos objetivos lo que la diferencia de las reuniones de grupo convencionales, es decir, de las reuniones sin estructura prefijada, que pueden tener un orden del día o no y donde la intervención del director de la reunión dependerá única y exclusivamente de sus cualidades y deseos personales.

Por ello es preciso evaluar lo que son capaces de aportar los grupos frente a los individuos en una situación de toma de decisiones. Esto ha sido motivo de profunda preocupación de los estudiosos de la materia en los últimos tiempos. En efecto, OSBORN ya en 1957 lo expresó al afirmar: «el ciudadano medio puede llegar a producir el doble de ideas cuando trabaja en grupo que cuando trabaja solo», lo que, traducido a términos coloquiales, vendría a querer decir: «uno más uno no son dos: es mucho más». Partiendo de estas investigaciones, es posible afirmar que se producen más ideas cuando las personas hacen un brainstorming en grupo que cuando lo hacen individualmente. Más aún, desde el exclusivo punto de vista de la producción de ideas, el brainstorming es superior, en situaciones de resolución de problemas, a las reuniones interactivas $\mathrm{y}$, ahondando aún más, es posible deducir con TAYLOR, BERRY y BLOCK que los grupos son superiores a los individuos en términos de producción de soluciones para resolver problemas.

Llegado a este punto resulta necesario precisar las características del grupo como factor determinante de la producción de ideas y los distintos matices que presenta la TGN frente a lo que se conoce como reuniones convencionales.

\section{EL GRUPO NOMINAL}

La evolución en este sentido, como ya quedó dicho, ha venido de la mano de OSBORN, que incidió en lo que llamó «el proceso de grupo nominal», donde los individuos trabajan en presencia de los demás pero escriben sus ideas por separado y no hablan sobre ellas. Los efec- 
tos positivos de este tipo de reuniones llaman la atención en relación con los resultados de una reunión convencional: se produce no sólo un mayor número de ideas, sino también un mayor número de ideas originales y éstas son de más calidad. En términos generales, se confirma que los grupos nominales, a la hora de generar información, son superiores a los grupos convencionales, ya que éstos dificultan el pensamiento creativo. El énfasis hay que ponerlo en la generación de ideas, pues, como afirman COLLAROS y ANDERSON (2), las inhibiciones individuales y las valoraciones prematuras dan como resultado un descenso sustancial de la creatividad y la originalidad.

\section{LAS REUNIONES CONVENCIONALES}

$\mathrm{Al}$ abordar este tema es inevitable reconocer el fracaso de las reuniones convencionales cuando a través de ellas se trata de efectuar un diagnóstico de una situación conflictiva. Las reuniones convencionales suelen ser improductivas, provocando sentimientos de frustración en los participantes que se traducen en cansancio y desgaste, tanto físico como intelectual, lo que produce ese estado de insatisfacción minuciosamente estudiado por los psicólogos. Y es que la mayoría de las veces, preguntados los asistentes a las reuniones acerca de su actitud negativa, afirmarán haber tenido la sensación de haber perdido el tiempo «girando alrededor de un círculo»-(un problema)—, como montados en una noria: se trata de las ocasiones en las cuales el «quid» de la cuestión ha quedado totalmente inexplorado. Otras veces, los invitados a la reunión dirán que babia muchos puntos que discutir pero que por algún extraño e inexplicable motivo todos volvian sobre lo mismo: «era como si el problema que figuraba como número tres hubiese estado cubierto de polvos mágicos y brillara con más luz que los demás... $\mathrm{Y}$, sin embargo, el punto primero era, evidentemente, el más importante».

También suele ser frecuente que esa sensación de frustración y cansancio a la que nos referimos se deba a procesos más complejos. Un análisis exhaustivo de una reunión media permite afirmar que en ella se «habla de muchas cosas», «se tocan muchos temas», incluso muchos más de los previstos en un orden del día, lo que puede resultar indudablemente creativo, pero sin olvidar que puede acabar conduciendo a una verdadera catástrofe: a mezclar problemas con soluciones, causas con efectos, estrategias con objetivos, de tal manera que todas las

(2) Collaros y anderson en el libro de Delbeco, Van de Ven y Gustafson, Group Tecb. niques for Program Planning. A Guide to Nominal Group and Delphi Processes, Ed. Green Briar Press, cap. 2, p. 17. 
ideas aparezcan como una nebulosa en la cabeza de los participantes.

Habrán sido los propios asistentes los que hayan aportado los ingredientes mientras que la reunión habrá funcionado a modo de «batidora». Lo anterior ha llegado a un extremo de máxima gravedad en alguna reunión donde alguien ha pretendido sorprendernos con su supuesta rapidez mental y ha ofrecido soluciones a un problema que todavía no babía sido planteado. Ello no sólo supone sobrevalorar la mente humana, sino introducir en el desarrollo de cualquier reunión un elemento de desorganización que es deseable evitar.

Finalmente, cabe aludir a ciertas situaciones de dominación por parte de algunos dentro de la organización y que no hacen sino contribuir a acrecentar el estado de desencanto de determinados sectores de la organización que se sienten meros comparsas que no son escuchados y a los que no les interesa participar en reuniones que son monopolizadas por el protagonismo de unos pocos.

\section{LA TGN COMO ALTERNATIVA}

Puede decirse que la TGN es fundamentalmente una técnica para generar ideas y resolver problemas. Desde el punto de vista de su objeto, tiene, pues, efectivamente, unas posibilidades de aplicación casi ilimitadas. Sin embargo, es preciso tener una visión algo más realista para decidir cúando y cómo debe ser utilizada para no aplicarla en temas de escasa trascendencia (3).

La TGN no es la solución que buscan los investigadores para los problemas de la reunión media o para las reuniones de rutina. Ha de ser considerada como un mecanismo de suma utilidad cuando:

a) El asunto a tratar en la reunión se considere de «extraordinaria importancia»o, como mínimo, «importante».

b) Se pretenda tener una visión lo más amplia posible de la cuestión.

c) Se trata de priorizar problemas o soluciones en el esquema de una reunión de tipo tradicional.

(3) Para hacernos una idea del significado de la anterior reflexión es suficiente con mencionar una recomendación de Alberta W. PARKER, profesora de la Universidad de Berkeley, California, hecha tras experimentar con la TGN: «La técnica no es una panacea para evitar todos los problemas que surgen en los procesos grupales. Deber ser utilizada con discriminación", en la Introducción al libro de DELBECQ, VAN DE VEN y GUSTAFSON, Group Techniques for Program Planning. A Guide to Nominal Group an Delphi Processes, Ed. Green Briar Press, p. 10. 


\section{OBJETIVOS DE LA TGN}

Es posible preguntarse, en este punto, cuál es la razón de que la estructura de la TGN sea precisamente ésta y no otra. Realmente, son los objetivos de la TGN los que la determinan, y sólo en función de ellos podrá ser comprendida. Los objetivos de la TGN pueden realmente resumirse en dos absolutamente genéricos, pero a la vez fundamentales: identificar y definir problemas, de una parte, y, de otra, mejorar el proceso de toma de decisiones.

Conseguir estos dos grandes objetivos de la TGN exige que las reuniones de grupo se organicen de tal forma que: a) aumente la productividad creativa de la acción grupal; $b$ ) se facilite la plasmación de las decisiones del grupo; $c$ ) se estimule la generación crítica de ideas; $d$ ) se apoye el proceso de agregación de opiniones individuales, y $f$ se aproveche la energía de los individuos integrados en el grupo.

\section{CARACTERISTICAS DE LA TGN}

Como ya hemos avanzado la TGN es una reunión de grupo estructurada para el análisis de problemas y la toma de decisiones.

El desarrollo de una TGN se produce en torno a la existencia de un problema que es preciso resolver y para lo cual se convoca a un grupo de expertos o conocedores de la cuestión, a quienes se solicita su colaboración de la siguiente manera: imaginemos una sala de reuniones donde hay de siete a diez personas sentadas de tal forma que todas pueden verse entre sí. La reunión se estructura en torno a un problema concreto, es conducida por un director que explica el alcance y desarrollo de la reunión, mientras que los participantes tienen asignada la tarea fundamental de anotar sus ideas. Al cabo de diez o quince minutos tiene lugar la formulación de ideas, que ha de seguir, por supuesto, una estructura. Cada individuo, por turno, presenta su lista de ideas al director, que va leyendo de viva voz y colocando en un panel situado a la vista de todos. En este punto, nadie discute. Las ideas van apareciendo mientras que los participantes escuchan. El producto de esta fase de la reunión será en realidad de dieciocbo a veinticinco ideas-propuesta. La votación posterior persigue una priorización de las ideas, de donde se extrae la decisión del grupo, que es la agregación de cada una de las propuestas individuales.

A partir de lo anterior, es posible deducir cuáles son las claves del proceso: $1 .^{\circ}$, la generación silenciosa de ideas por escrito; $2 .^{\circ}$, la agrupación de las ideas de los participantes en el panel; $3 .^{\circ}$, la discusión de cada una de las ideas para su clarificación, y $4 .^{\circ}$, las votaciones individuales que priorizan las ideas. 
Este proceso no basado en mecanismos interactivos presenta, pues, las siguientes características:

1. Permite la exposición ordenada de las ideas.

2. Favorece las manifestaciones sintéticas.

3. Recupera las posiciones minoritarias.

4. La aceptación de las ideas por el grupo no depende de la brillantez de su exposición.

5. El producto resultante es la opinión de un colectivo.

6. Permite un debate cualitativo final.

7. Asegura la participación de todos los asistentes.

8. Incorpora técnicas de voto que permiten priorizar la opinión del grupo.

\section{DESARROLLO Y FASES DE UNA TGN}

\section{ELEMENTOS QUE INTERVIENEN EN LA TGN}

\subsection{El Director de la TGN}

En la TGN es fundamental la figura del Director. El Director es un elemento crítico. Así, los expertos afirman que el Director de la TGN debe tener unas dotes determinadas para que la reunión sea eficaz. Y es que no se trata de dirigir una reunión de tipo convencional donde el líder puede ejercer un tipo de influencia determinada y conducir con una cierta habilidad a los participantes a su terreno. El Director de la TGN es un líder con una misión especifica. De él depende todo el proceso TGN, es decir, que se verifiquen cada una de las fases y que se cumplan los objetivos de la sesión. Téngase en cuenta que la TGN es, entre otras cosas, una reunión sin orden del día y que los participantes al ser convocados es posible que desconozcan totalmente la finalidad de la reunión salvo el tema de que van a tratar.

En la TGN, más que en cualquier otra reunión, los resultados dependerán de las cualidades del Director. Para ello, se afirma que es definitivo lo siguiente: que el Director comprenda perfectamente el proceso y que sea uun convencido" de las posibilidades de la TGN. Es importante también que tenga suficiente confianza en sí mismo para conducir al grupo a través de la TGN. Hay que considerar la posibilidad de que la mayoría de los asistentes jamás hayan participado en un proceso de grupo de semejantes características y, por ello, en los primeros momentos muestren una cierta desconfianza, incluso hostilidad.

Para afrontar el inconveniente anterior, es recomendable que el Director sepa muy bien lo que se trae entre manos, ya que probablemente no será suficiente con su actitud para convencer al grupo de las bon- 
dades de la TGN, sino que será necesario que tenga una cierta legitimación «moral» ante el grupo para que éste confíe en él. En este mismo sentido, es aconsejable que el Director sea un «experto en TGN» $\mathrm{y}$, en segundo término, que sea un buen comunicador. Es importante que, en un momento dado, los participantes lleguen a comprender el objetivo de la TGN con absoluta exactitud, y ello deberá haberles sido transmitido por el Director. Si esto lo bace con eficacia ya no necesitará justificar cada uno de los pasos del proceso y éste será mucho más fluido $\mathrm{y}$, por tanto, rápido. -...

\subsection{Participantes}

Otro de los aspectos importantes a la hora de celebrar una TGN es precisamente el de los invitados. En definitiva, se trata de otro «factor crítico», pues, como correctamente dicen VAN DE VEN y DELBECQ, «la TGN funciona como una aspiradora: es un potente medio de absorber toda la información que poseen los miembros de un grupo. Sin embargo, si no hay nada que extraer, aun una aspiradora potente resulta totalmente inútil». Lo primero es, pues, determinar la materia de que se trate, para llevar a cabo, en segundo término, una aproximación al tipo de miembros de la/s organización/es que puedan tener la información que se desea. Lo siguiente será valorar la actitud personal de los participantes en la hipotética reunión, así como su disponibilidad para formar parte de ella.

Por lo que se refiere a los participantes, es necesario sopesar hasta qué punto es conveniente convocar a un grupo bomogéneo o beterogéneo de miembros. Es cierto que los grupos heterogéneos son más creativos, pero en ellos puede haber problemas de comunicación. Puede suceder que la búsqueda de la creatividad suponga invitar a personas que ocupen puestos en toda la escala de la organización o incluso provenientes de sectores y ambientes absolutamente dispares. Ello implicaría que algunos participantes se sintieran cohibidos ante la presencia de personas determinadas, lo que sucedería frecuentemente si la TGN se realizara con los miembros de una organización concreta y a ella se invitara al «jefe», y aunque en la TGN su carácter de «grupo nominal» minimiza este problema, conviene tenerlo en cuenta.

Nos recuerdan una vez más los creadores de la TGN que, en todo caso, será preciso que los participantes sean capaces de comunicarse en lo que ellos llaman «un lenguaje común», lo que no significa en absoluto que los invitados tengan que tener la misma opinión, la misma perspectiva o el mismo punto de vista sobre el problema, pero sí, al menos, que estén situados dentro de una determinada «escala cultural» para que, en la fase de clarificación de ideas, nadie se sienta fuera de lugar. 
Es determinante en una TGN valorar hasta qué punto los participantes necesitan de una determinada preparación psicológica. Los asistentes a una TGN realizan un esfuerzo psíquico y físico considerable, que el Director debe tener en cuenta en todo momento.

Paralelamente, una TGN es un proceso pensado para un número determinado de participantes. Lo ideal es que participen siete personas, aunque también es posible que la técnica funcione cuando intervenga un número de miembros que puede estar entre cinco y nueve. Cuando es preciso recibir información de más de nueve personas se hace necesario considerar si es preferible celebrar dos sesiones o una sesión en la que participen varios grupos simultáneamente, en cuyo caso, el Director deberá contar con la colaboración de uno o varios ayudantes.

\subsection{Preguntas a formular}

Otro aspecto a tener en cuenta es la formulación de las preguntas. Efectivamente, hay que plantear unas buenas preguntas para que la $T G N$ pueda surtir efectos. Si «una buena pregunta es aquella con la que se obtiene el tipo de respuestas que se buscan», resulta imprescindible formular las preguntas tras un período de reflexión que permita evaluar la validez de las mismas. Para contrastar lo anterior, el grupo de trabajo encargado de preparar la TGN discutirá, en primer lugar, el objetivo de la reunión. Una especificación precisa de ello es determinante para formular una buena pregunta. Una vez hecho esto, el equipo profesional determinará el tipo de respuestas que se aspira obtener de los participantes, especialmente en términos de abstracción/especificidad y profundidad/amplitud. Ello se hará experimentando con la pregunta en un grupo piloto, formulando preguntas alternativas y utilizando cualquier otro sistema de similares características.

\subsection{Votaciones}

La información que proporcionan los asistentes tiene un valor determinado, pero deberá priorizarse. El sistema de votación es otro factor clave en una TGN. El cómo se llevan a cabo las votaciones será determinante a la hora de sacar partido de la información. La elección del sistema de votación es, pues, un elemento que condicionará el éxito de la Técnica. Un estudio previo de las distintas posibilidades de los sistemas de votación será, por tanto, fundamental, así como el elemento clave de que las votaciones sean secretas. 


\section{TAREAS PREPARATORIAS}

El Director debe asegurarse de que antes de comenzar la técnica se verifican las siguientes tareas preliminares:

a) elección y preparación de la sala donde se va a llevar a cabo la reunión;

b) aportación de los medios materiales necesarios;

c) presentación de la TGN.

\subsection{La sala de la reunión}

La mayor parte de las actividades de la TGN suceden en relación con un grupo pequeño de personas. El Director debe, por tanto, elegir una sala que sea suficientemente espaciosa para que los participantes pueda colocarse en torno a una mesa en forma de «U». Sin embargo, la sala no debe ser excesivamente grande para el número de asistentes, ya que tal circunstancia actúa negativamente sobre la cohesión del grupo y hace más difícil la concentración y creación de un ambiente de trabajo.

La sala debe permitir disponer de una pantalla y retroproyector y paredes útiles para la colocación de tantos paneles como preguntas se vayan a formular al grupo.

\subsection{El material}

En una TGN se ha de contar con lo siguiente:

a) Paneles de corcho en donde situar las cartulinas (tantos paneles como preguntas se vayan a formular). (De 2 a $2,30 \mathrm{~m}$. de largo por 0,80 a $1 \mathrm{~m}$ de ancho.)

b) Un papelógrafo.

c) Cartulinas de 21 centímetros de largo por 15 centímetros de ancho (a poder ser autoadhesivas).

d) Rotuladores para cada participante.

e) Cámara Polaroid para obtener fotografías de los paneles una vez formuladas todas las ideas.

f Retroproyector y pantalla (para apoyar con transparencias la explicación del proceso TGN).

Las TGN funcionan sobre la base de que las ideas de los participantes puedan mostrarse por escrito al grupo en su conjunto, lo que 
significa que resulta imprescindible un panel donde se recojan las ideas escritas y un rotafolios donde se reflejen las ideas claves aparecidas.

\subsection{Explicación de objetivos}

Cuando un grupo de individuos se enfrenta a un trabajo en común es necesaria una explicación acerca de la importancia de su tarea conjunta. El producto del grupo será de tanta más calidad cuanto mejor se haya percibido lo anterior. Por ello, es importante que el Director explique los objetivos del grupo en su conjunto y el rol de cada uno de los miembros como participantes individuales, con el fin de romper las barreras que puedan existir entre ellos y facilitar la comunicación.

Lo anterior significa que en la explicación inicial de objetivos:

- se resaltará la importancia de la labor del grupo;

- se resaltará la importancia de las aportaciones de cada participante;

- se hará una referencia a la utilidad de los resultados de la reunión y al valor de éstos para poder adoptar decisiones.

\section{DesarRollo del PROCESO}

En este punto nos referiremos al desarrollo de una sesión que contará con seis o siete participantes y un Director que tiene la misión de formular las preguntas que se van a someter al grupo (no más de tres en una sesión) y para cada una de cuyas respuestas se exigirá respetar el siguiente proceso:

\subsection{Generación silenciosa de ideas por escrito (Fase $1 .^{a}$ )}

El primer paso una vez efectuada por el Director la explicación de los objetivos de la reunión y del funcionamiento de la TGN consistente en que los participantes formulen sus ideas por escrito en silencio y de forma independiente. Para ello el Director llevará a cabo las siguientes actividades:

- presentará por escrito al grupo la pregunta que encabezará el panel correspondiente;

- leerá la pregunta en voz alta;

- pedirá a los participantes que escriban sus ideas sobre la cuestión formulada en frases cortas, con letra mayúscula y clara utilizando los papeles autoadhesivos o cartulinas preparadas a tal fin; 
- pedirá a los participantes que trabajen en silencio y de forma independiente;

- moderará la reunión para que la conducta de los participantes se ajuste a la técnica, otorgándoles un tiempo determinado (entre cinco o diez minutos) para que presenten sus ideas por escrito (a veces el Director de la TGN puede recomendar que se formulen un mínimo de ideas o respuestas por cada una de las preguntas que se efectúen).

El Director procederá a repartir las hojas de trabajo a los participantes. Una vez hecho esto, y transcurrido el tiempo previsto, recogerá todas las hojas utilizadas y comenzará a colocarlas en el panel correspondiente a la primera pregunta.

\section{Las claves de la Fase 1."}

Los principales puntos que el Director de la TGN deberá tener en cuenta en esta fase son:

- no detenerse en aclarar cuestiones que no se refieran al proceso en sí;

- presentar la pregunta por escrito;

- conseguir una buena conducta de grupo y que los participantes escriban en silencio.

También es importante tener en cuenta que una vez que se presenta la pregunta por escrito, el Director debe evitar hacer aclaraciones sobre la interpretación de la misma, ya que si lo hace, podrá condicionar las opiniones del grupo.

Cabe la posibilidad de escribir la pregunta directamente en el papelógrafo, en vez de situarla encima de cada panel, aunque es mucho más eficaz esta última opción, ya que contribuye a que los miembros del grupo interioricen la vinculación existente entre preguntas e ideasrespuestas y esto es especialmente importante en el caso de diseño de estrategias, toma de decisiones, etcétera.

\subsection{Registro de ideas (Fase $2 .^{a}$ )}

La segunda fase de la TGN consiste en recoger y colocar sobre el panel las ideas de cada uno de los participantes de tal forma que se vayan agrupando aquellas ideas de contenido idéntico $u$ homogéneo. Ello significa que el Director de la TGN irá alrededor de la mesa, recogiendo las cartulinas de cada participante, para situarlas en el panel, 
previa lectura del contenido de cada una de ellas en voz alta dirigiéndose al grupo.

Las ventajas de registrar la información de esta manera son las siguientes:

- se obtiene un aceptable grado de anonimato: separación ideas/personas;

- se posibilita manejar un gran número de ideas;

- permite considerar conjuntamente ideas en conflicto y agrupar las que tienen idéntico contenido;

- se posibilita la adhesión a las ideas formuladas por otros;

- se consiguen unos resultados por escrito, que se generan en un espacio muy corto de tiempo.

Al participar todos los miembros del grupo, se incrementa la creatividad, mientras que.la lectura en voz alta por el Director de todas las ideas presentadas permite resaltar la importancia de las ideas por encima del autor de las mismas.

La formulación escrita de las ideas, y su integración en el panel, aparece como más objetiva y menos personal que un comentario hablado. Por otra parte, si la idea está escrita manteniendo un cierto anonimato resulta más sencilla separarla de la persona de quien proviene.

En esta fase el papel del Director de la TGN adquiere una importancia crucial, ya que de su conocimiento y sobre todo de su habilidad en la dirección se deducirá un alto grado de consenso en la elaboración del panel. Es en este momento de identificar y agrupar las distintas ideas cuando recabará del grupo su opinión, de suerte que el conjunto de ideas que se contiene en el panel se agrupen con el consenso de todos en tantas alternativas o ideas distintas como sea necesario, haciendo para ello un esfuerzo de síntesis. En este punto, y ante la dificultad de definir el contenido real de una idea, el Director puede incluso solicitar que se identifique el autor de la misma si es que el grupo en la discusión la está interpretando de forma incorrecta.

No hay que olvidar tampoco la importancia que tiene el ir pidiendo a los participantes que expresen sus opiniones. Con ello, el Director consigue deshacer las inhibiciones individuales y la integración del grupo.

Finalmente, un panel con las ideas agrupadas puede considerarse como una recompensa de primera mano del trabajo en equipo. Los participantes suelen quedan verdaderamente impresionados por el flujo de ideas que el grupo es capaz de generar.

Es preciso valorar también el consenso que se obtiene al agregar ideas coincidentes, e igualmente, los diferentes resultados que se obtienen si se consideran por separado las ideas de cada individuo o si se consideran éstas en su conjunto. 
Esta fase es la clave de la TGN, y de ahí su importancia. Una vez agrupadas las ideas en el panel integrándolas en círculos que claramente las diferencien de otras ideas, el Director procederá a sintetizarlas en conceptos que irá escribiendo sobre el mismo panel o en el papelógrafo de forma clara con la finalidad de facilitar al grupo la visualización del conjunto de alternativas que se han producido a la pregunta formulada.

Las claves de la Fase 2."

De acuerdo con lo expuesto en esta fase el éxito de la misma requiere por parte del Director:

- anunciar claramente los objetivos que se pretenden;

- pedir que se presenten las ideas en frases cortas;

- reflejar todas las ideas emitidas (sin omitir ninguna);

- confirmar que es deseable que se enuncien variaciones sobre una misma idea;

- situar las ideas sobre el panel;

- agruparlas de acuerdo con el contenido en alternativas diferentes, para ello es recomendable rodear con un rotulador el conjunto de cartulinas que contengan ideas semejantes para efectuar una separación gráfica entre las distintas alternativas;

- suscitar aclaraciones del grupo sobre cómo efectuar la agrupación;

- hacer la síntesis de las ideas expuestas en el papelógrafo o sobre el mismo panel;

- procurar que en todo momento el panel con todas las ideas sea visible para todo el grupo, así como la síntesis del papelógrafo.

Hay que tener en cuenta que el Director debe medir bien los tiempos evitando que la fase de aclaración del grupo se alargue más de lo imprescindible.

\subsection{Debate aclaratorio (Fase 3.')}

La tercera etapa de la TGN consiste en aclarar por turno las ideas expuestas tanto respecto a su contenido como a su agrupación en ideas homogéneas.

La discusión supone abrir un debate aclaratorio de las ideas que aparecen tanto en el panel como en el papelógrafo. El Director señala la idea número 1, la lee en alto y se dirige al grupo para constatar si hay alguna pregunta o si es preciso hacer alguna aclaración y les pide 
que manifiesten su acuerdo o desacuerdo con ella. El Director concede un tiempo para la discusión y procede a señalar el ítem número 2 , y así sucesivamente.

Hay que tener en cuenta que el objeto fundamental de la discusión es aclarar los conceptos para que todos los participantes acaben comprendiendo el significado de las palabras y de las frases expuestas en el panel. Efectivamente, el fin de la discusión es aclarar las ideas expuestas en el papelógrafo, pero a la vez se trata de minimizar la influencia que puedan ejercer los participantes más «importantes»o más brillantes didácticamente sobre el resto del grupo.

Para lograr este doble objetivo el Director ha de tener en cuenta:

- que la discusión no se debe centrar en una única idea o conjunto de ideas;

- que no se debe desembocar en una discusión general sobre la pregunta.

La finalidad de la discusión es proporcionar un período de reflexión para el análisis de las ideas, pero no se trata de resolver las diferencias de opinión, que en todo caso se pondrán de manifiesto en la votación. En este punto conviene recordar que esta técnica no funciona como mecanismo interactivo, sino que se apoya en el grupo para poder efectuar una priorización de las ideas expuestas mediante una votación.

Las claves de la Fase 3."

De acuerdo con lo manifestado el Director debe hacer especial hincapié en:

- explicar a los participantes el contenido de la fase en cuestión;

- conducir el debate para evitar que a algunas ideas se les dedique demasiado tiempo en detrimento de otras;

- evitar que en este período de aclaraciones los dominantes del grupo traten de hacer un panegírico de las ideas expuestas.

Finalmente, hay que tener en cuenta que no se debe pedir a los participantes que aclaren sus propias ideas, a no ser que voluntariamente lo quieran hacer. A pesar de que la mayoría de ellos clarificarán sus ideas voluntariamente es necesario dejar bien claro que esta labor es una tarea de grupo y no la responsabilidad unilateral del autor de la idea. 


\subsection{Votación para la priorización de las ideas (4) (Fase 4. ${ }^{\circ}$ )}

En la reunión media TGN se producen alrededor de tres ideas por pregunta formulada y por cada miembro del grupo durante la fase de generación de ideas. A través de la discusión que se suscita una vez completado el panel, los miembros del grupo pasan a esclarecer el significado de cada idea, y a agruparlas en ideas homogéneas. De alguna forma, sin embargo, el grupo tiene que agregar sus opiniones con el fin de determinar la importancia relativa de cada una de las ideas individuales.

Las teorías sociopsicológicas han hecho grandes esfuerzos para determinar el mecanismo apropiado para agregar las opiniones de grupo. Se ha demostrado que el siguiente método es el que más exactamente refleja la decisión del grupo sobre sus propias preferencias. Se trata de lo siguiente:

- los participantes deben expresar sus opiniones individuales;

- las opiniones individuales deben plasmarse matemáticamente (categorización o priorización de ideas);

- el valor de la medida $(\mathrm{x})$ de las opiniones independientes debe tomarse como la decisión del grupo.

Este método ha sido utilizado eficazmente como procedimiento de votación en las reuniones $T G N$, valorándose muy positivamente en comparación con los sistemas convencionales alternativos que existen para llegar a una decisión de grupo: consenso, la regla de la mayoría y las listas independientes. Ello pone de manifiesto el valor de este simple método matemático. En suma, se puede incrementar la exactitud de las opiniones haciendo que los participantes expresen sus opiniones individuales en términos matemáticos.

Las claves de la Fase $4 .^{2}$

La última fase de la TGN dirigida a obtener una priorización de las ideas expuestas presenta como puntos claves para el Director:

- Pedir al grupo que seleccione de la lista completa de ideas síntesis del papelógrafo, un número concreto de ítems que serán los más importantes.

(4) La mecánica de la votación admite diversas soluciones, si bien la presentada en el presente artículo responde a una adaptación del modelo clásico efectuada por la IGSAP en sus actuaciones de consultoría por considerarla mucho más rápida y tan operativa como cualquier otra. 
Para ello se cuentan y el Director otorga a cada participante un número de votos que suele ser alrededor de la mitad de alternativas que han resultado y que deben ser objeto de priorización.

- Solicitar a los miembros del grupo que piensen cómo distribuir esos votos.

Para ello se recomienda que traten de discriminar las alternativas a votar, dando a unas más votos que a otras, aunque cualquier mezcla que hagan será útil (por ejemplo, si se tienen seis votos por persona, se pueden distribuir así: 3,2 y 1 ó $3,1,1,1$, 0 ...). A continuación se les da tiempo para que reflexionen y piensen cómo van a utilizar los votos. Este período debe ser corto, para lo cual se va preguntando al grupo si ya todos han tomado su decisión. Cuando esto se ha producido, se pasa a efectuar la votación propiamente dicha.

En esta etapa se trata de conseguir una votación que sea muy rápida y lo más «secreta» posible. Si se utiliza una urna, la votación es secreta pero el recuento es muy lento. Por ello es preferible repartir pequeñas pegatinas de colores (circulares, cuadradas, etc., pero del tamaño de una moneda), y para que el voto sea lo más reservado posible, se procura que todos voten simultáneamente. Para ello se pide a todos los miembros del grupo que cojan sus votos, se levanten y se acerquen a un metro y medio del panel. Cuando están todos colocados proceden a votar (pegar sus votos sobre las agrupaciones de ideas que les parezcan más adecuadas) todos a la vez. De esta manera la rapidez otorga cierta discreción a las votaciones, y aunque el secreto no puede garantizarse la situación es aceptable, y además nos permite pasar a la etapa siguiente con rapidez.

Una vez acabada la votación, todos los miembros del grupo vuelven a sus lugares, procediendo el conductor de la TGN a contar los votos de cada alternativa y a recoger por escrito el resultado sobre el panel.

Habitualmente lo significativo no es la comparación entre las cifras absolutas de votos obtenidos, sino el orden de preferencia que se ha obtenido (otorgando el 1 al más votado, el 2 al siguiente y así sucesivamente), así como la carga relativa de votos recibida.

Finalizada la votación se concluye la fase de la TGN correspondiente al primer panel, iniciándose de nuevo el proceso cuando el objeto de la reunión sea obtener respuestas a varias cuestiones.

En algunos supuestos la reunión TGN puede darse por finalizada en esta Fase 4. En otros casos, donde es precisa una mayor exactitud en las votaciones, se pueden añadir las dos siguientes fases: Discusión de la votación preliminar y Segunda votación o votación definitiva. 


\subsection{Discusión de la Votación preliminar (Fase 5.")}

Se trata de una fase breve que puede incluirse en el proceso para añadir más exactitud a los resultados que se han anotado en el papelografo. El propósito de esta posible discusión es el siguiente:

- examinar esquemas de voto incongruentes;

- dar la oportunidad de volver a discutir ideas que hayan recibido muchos o muy pocos votos.

Aunque de esta segunda discusión antes de volver a votar pocas veces se derivan cambios radicales, a veces sí conduce a unos resultados más exactos.

Las claves de la Fase 5."

En esta fase el Director debe:

- explicar que el objetivo es clarificar algunas cuestiones necesarias;

- asegurarse de que la discusión sea breve, para no distorsionar los resultados, ya que existe la posibilidad de que en esta fase algunas ideas no se discutan en absoluto.

Los estudios sobre votaciones demuestran que el proceso en tres etapas: votación, discusión y segunda votación, ofrece unos resultados bastante exactos sobre las preferencias del grupo en relación con lo que pudiera ofrecer una votación única.

\subsection{Segunda votación o votación definitiva (Fase $6^{a} .^{\text {) }}$}

La Fase 6." es la etapa final de la TGN. Esta votación consiste en combinar las opiniones individuales para formar una opinión de grupo. La votación final:

- Determina los resultados de la reunión.

- Hace que los participantes tengan la sensación de haber terminado el trabajo.

- Proporciona un documento sobre la opinión del grupo.

Aquí es posible seguir el mismo procedimiento de votación que en la votación preliminar. Pero también se pueden utilizar técnicas de votación más exactas. El utilizar o no estas técnicas depende del grado de exactitud que se desee en los resultados, de la materia objeto de 
investigación y de la cantidad de información que el grupo posea. En general, podrá procederse a colocar las preferencias de los participantes en escalas de 0 a 10 o en escalas continuas. Si es preciso considerar la magnitud de las diferencias entre prioridades, será necesario utilizar el método de escalas. Sin embargo, si la materia es muy general será suficiente con volver a utilizar el sistema de la votación preliminar.

Una vez finalizada la Técnica, el Director cuenta con una información de primera línea sobre el asunto tratado en la reunión. El Director debe, para su correcta utilización, elaborar un Informe en donde deberán describirse todos los pormenores de la realización de la Técnica. En la distribución del Informe se deberá necesariamente incluir a todos los participantes, ya que ello aumentará considerablemente su predisposición a colaborar en futuros procesos de grupo.

\section{VENTAJAS DE LA TGN}

A lo largo de esta exposición han ido apareciendo los aspectos positivos de la TGN, pero haciendo un esfuerzo de síntesis se podrian distinguir ventajas de distinto tipo:

A) Creativo, en cuanto que permite:

- La proliferación de ideas.

- La mayor calidad de su contenido.

- Una presentación sintética de las mismas.

B) Participativo, en cuanto que:

- Todos participan.

- No se impone un lider.

- Se evita el gregarismo.

- No permite la lucha por conseguir una buena imagen personal.

C) Operativo, en cuanto que:

- Se obtienen resultados concretos.

- Se evitan tensiones en el grupo.

- Se mantiene al grupo centrado en la tarea.

- No se producen interrupciones.

Si tuviéramos, no obstante, que subrayar la esencia de esta técnica, podríamos decir que a través de ella las ideas se imponen a las personas para ser asumidas por el grupo por lo que valen en sí mismas.

En este sentido conviene subrayar dos aspectos de la TGN: el papel del Director y la neutralización de los efectos negativos del liderazgo. 
En primer lugar, nos referimos al papel concreto del Director. El Director en una TGN es un elemento clave por lo que a la conclusión del proceso se refiere. Sin embargo, desde el punto de vista del contenido de los resultados, su persona o, mejor dicho, su personalidad, su forma de opinar, su status en la organización, etc., resultan a todas luces irrelevantes. Lo que queremos decir con esto es que la TGN ataca frontalmente el «dirigismo» que demasiado frecuentemente ejercen en las reuniones sus presidentes. Si las instrucciones que da el Director durante la celebración de la Técnica son, como deben ser, distanciadas y asépticas, entonces resultará difícil pronosticar las ideas que irán apareciendo en el panel. Incluso, en ocasiones, los resultados serán «desconcertantes». Y esto, ¿es bueno? Lo es, en la medida en que significa que a los asistentes a una TGN no sólo se les pide su opinión, sino que también se les permite que la expresen libremente. En una TGN, el Director puede participar activamente como un miembro más del grupo o no; podrá expresar sus ideas o no. Pero, si lo hace, tendrá idénticas posibilidades, en términos de conseguir votos, que el resto de los miembros del grupo.

En segundo lugar, suele suceder también que las reuniones interactivas acaban siendo dominadas por determinados individuos, que bien por el puesto que ocupan en la organización o bien por sus dotes personales, son capaces en un momento dado de asumir el liderazgo. Ello tiene el fundamental inconveniente de que «inexplicablemente, las ideas por ellos propuestas son las más votadas».

En una TGN cabe la posibilidad de que las personas de más status o las extrovertidas acaben «hablando más» que el resto del grupo en la fase de discusión de las ideas, pero ello quedará compensando por las votaciones independientes y secretas a ideas concretas y por la brevedad que deberá caracterizar a sus intervenciones. Así, será posible afirmar que en la TGN no se ve claramente la relación entre «cantidad bablada» e influencia en el resto del grupo, porque los miembros más introvertidos o de menor status puede que no estén dispuestos a expresar sus tesis de viva voz y a defender sus ideas hasta el final, pero sí votarán de forma independiente.

Es necesario puntualizar, por último, que la TGN puede utilizarse independientemente del nivel cultural de los participantes. Realmente, la experiencia confirma que una TGN puede llevarse a cabo haciendo participar a los miembros del Consejo de Dirección de un Departamento Ministerial, pero la convocatoria a una TGN puede ir dirigida también a personas que no estén habituadas a escribir. De igual modo, en una TGN no se necesitan dotes de gran orador para sacar adelante las propias ideas. Si una idea es buena, recibirá normalmente votos sólo por el becho de serlo. 


\section{INCONVENIENTES DE LA TGN}

Los inconvenientes de una TGN no son realmente tales, sino más bien factores a tener en cuenta a la bora de llevarla a cabo. La TGN es un proceso grupal no interactivo con un objetivo especial, por lo que resulta necesario especificar las circunstancias en las que deber ser utilizado.

En primer lugar, la TGN sirve tanto para identificar los elementos de una situación problemática como para formular un programa de soluciones $\mathrm{y}$, en segundo término, permite establecer prioridades.

En consecuencia:

\section{1. $\quad$ No debe utilizarse indiscriminadamente}

En este mismo sentido, como ya he quedado dicho, la TGN no es la solución de la reunión media. Sólo aquellos asuntos clasificados como «importantes» o que, por algún motivo, se consideren de especial trascendencia para la organización deben pasar por el filtro de una sesión de TGN. En esta misma línea, los problemas con una estructura especialmente compleja, aquellos que tengan derivaciones o consecuencias incalculables o aquellos que afecten a diferentes sectores pueden ser perfectamente analizados con el apoyo de la Técnica. Como textualmente dicen DELBECQ y VAN DE VEN, «la TGN no debe utilizarse para resolver problemas simples». La TGN supone un desgaste considerable de los medios personales y por ello antes de proceder a su aplicación en una situación determinada se debe calcular si es precisa toda la información que se obtiene a través de ellas. Si ésta no es necesaria, el mecanismo TGN se deberá obviar.

\section{Puede producir rechazos}

En ocasiones sucede que los individuos sienten un rechazo inexplicable a los procesos de grupo estructurados. Los psicólogos nos recuerdan que en este tipo de situaciones es probable que algunas personas se sientan cohibidas a la hora de escribir sus ideas o a la hora de votar. Puede que se sientan manipuladas por todo lo que supone la Técnica en sí, por la amenaza de algún miembro presente en la reunión que aspire a «dominar» la situación o incluso por la actuación de un Director que simplemente no le inspire confianza.

También puede suceder a la inversa, que los individuos con necesidad de dominar o con una posición alta en la organización se sientan 
amenazados por la Técnica, al percibir que les va a resultar imposible controlar el desarrollo de la reunión. Sus reservas a la Técnica se desvanecerán, sin embargo, cuando hayan experimentado con ella y hayan podido comprobar la alta calidad del producto.

\section{Exige contar con un Director capacitado}

Si hemos dicho que la Técnica gira en torno a la figura del Director, ello significa que gran parte del éxito o del fracaso de la sesión podrá atribuirsele a él. El Director tendrá que sopesar lo que supone plantear una buena pregunta TGN y sobre él caerá la responsabilidad de que los participantes den aquellas respuestas que se buscan. Para lograr lo anterior, tendrá que consagrar tiempo y esfuerzos a meditar sobre la posible pregunta, a experimentar con ella con grupos piloto y a plantear en su caso preguntas alternativas. Durante la Técnica en sí, el Director deberá tomar una determinada actitud de la cual se pueda deducir que tiene las dotes necesarias para conducir al grupo a través del proceso, demostrando que tiene una confianza en sí mismo basada en su propia legitimidad moral ante el grupo, lo cual implica una actitud prepontente. Durante todo el proceso, el Director deberá valorar las diferentes capacidades de los participantes, tanto intelectuales como de expresión, para sacar el máximo partido de la situación y deberá conseguir que ningún participante domine la reunión.

\section{4." Aceptación de sus resultados}

Con todo y con eso, puede que el principal punto débil de una TGN sea comun a todo proceso grupal. Las conclusiones a las que se llega después de una TGN son, desde el punto de vista de su naturaleza, esencialmente idénticas a las que surgen en cualquier otra reunión de grupo. Estas tienen que ser juzgadas desde un doble ángulo: el de su aceptación y el de su calidad.

Desde el punto de vista de su aceptación, habrá que tener en cuenta distintas variables que influyen en ella. Por ello, los resultados de una TGN a los ojos del resto de la organización o de los grupos que no han participado en el proceso puede que en un principio no tengan más aceptación que los resultados de cualquier otro proceso grupal.

Sin embargo, pensemos por un momento en esas variables que influyen en la aceptación. Una de las más importantes es la calidad. $E l$ output de una TGN es cualitativamente superior al de cualquier otro proceso grupal. La calidad del producto es la clave de la TGN. En un mo- 
mento donde las empresas y organizaciones en general buscan el camino que les conduzca al concepto de Calidad Total, cualquier método que suponga añadir algo en ese sentido debe ser tenido en cuenta en el proceso de toma de decisiones.

\section{FACTORES DE EXITO DE UNA TGN}

En la TGN, según ha quedado expuesto en los epígrafes anteriores, concurren ventajas e inconvenientes que es preciso tener presente para conseguir una utilización eficaz y eficiente de la misma. Sin embargo, existen factores que por sí mismos van a ser determinantes del éxito o del fracaso de esta técnica. A modo de conclusión pueden enumerarse como factores necesarios para el éxito de una TGN los siguientes:

1. Una situación que exija la aplicación de la TGN.

2. Un Director que utilice correctamente la técnica.

3. Una formulación adecuada de las preguntas.

4. Una selección acertada de los asistentes.

5. Una explicación correcta de los objetivos y desarrollo de la reunión.

6. Disponer de los medios materiales imprescindibles para su realización.

\section{IMPLANTACION EN EL SECTOR PUBLICO}

La modernización de la Administración Pública pasa necesariamente por la incorporación a su Sistema de Dirección de un conjunto de técnicas gerenciales, que si bien surgen en el sector privado como instrumentos para mejorar la eficacia de la organización, pueden ser asumidas, adaptadas y aplicadas con éxito por la Administración, fundamentalmente en este momento en que la Administración, al formular sus estrategias de cambio a medio y largo plazo, se apoya en la obtención de resultados concretos como respuesta a la creciente demanda de eficacia de los ciudadanos.

La TGN constituye una herramienta de trabajo apta para ser utilizada en el sector público toda vez que es rentable en términos tiempo-resultados.

La Administración pública como el resto de las organizaciones complejas funciona apoyándose fundamentalmente en la toma de decisiones que generalmente se produce tras largas e infructuosas reuniones de consejos, comités, grupos de trabajo, etcétera. 
La toma de decisiones sobre problemas relevantes de las organizaciones, como pueden ser la fijación de estrategias, la definición de objetivos, la detección de problemas, la elaboración de alternativas, el diseño de políticas sectoriales concretas, etc., son actuaciones que requieren un previo análisis de la situación como requisito para elaborar o proponer soluciones. En este marco de actuación, en el que es preciso efectuar diagnósticos de precisión, es donde la Administración puede rentabilizar al máximo la utilización de una técnica como la descrita utilizando medios propios.

La obtención de unos resultados rápidos, como permite obtener esta técnica, sitúa a la organización que la utiliza en condiciones óptimas para abordar la solución de problemas en tiempo real, lo que aumentará su grado de eficacia al ofrecer diagnósticos y soluciones para resolver problemas trascendentes y relevantes para la misma.

\section{EXAMEN DE UN CASO PRACTICO}

La descripción de esta técnica se complementa con la presentación de un supuesto práctico desarrollado en el mes de mayo de 1990 y que constituye una clara muestra de cómo una reunión de cuatro horas de duración puede alumbrar diagnósticos y propuestas de gran calidad con la finalidad de ofrecer un apoyo técnico a quien debe adoptar una decisión.

\section{ANTECEDENTES}

La Subcomisión de Planificación del Plan de Formación constituida en el INAP, como consecuencia de lo previsto en la Resolución de 14 de febrero de 1990, que creó la Comisión Consultiva para la elaboración del Plan de Formación del Instituto, acordó dentro de su plan de trabajo recabar de la Inspección General de Servicios de la Administración pública la realización de dos TGN (Técnica del Grupo Nominal) con la finalidad de obtener una opinión cualificada de un grupo de expertos en torno a la problemática actual de la formación del personal al servicio de las Administraciones públicas.

\section{ObjetTvos}

La Subcomisión de Planificación del Plan de Formación se planteó, en la reunión celebrada el 19 de abril, la necesidad de realizar una labor de prospección que permitiera realizar un análisis cualitativo y 
cuantitativo de la distancia existente en la actualidad entre oferta y demanda.

Para abordar estas cuesiones se acordó en el seno de la Subcomisión una doble vía de actuación, consistente en la preparación de sendas encuestas dirigidas a los responsables departamentales de la Formación y en la utilización de la Técnica de Grupo Nominal, como instrumento complementario, para obtener una respuesta cualificada de un grupo de expertos.

Para la realización de la TGN se discutieron y aprobaron en la sesión del día 3 de mayo tanto la lista de expertos a participar como las cuestiones que se debían formular y cuya contestación debería permitir:

1. Identificar los aspectos positivos que se derivan del actual sistema de formación.

2. Detectar los problemas más significativos y disfunciones existentes en la actual política de formación.

3. Alumbrar alternativas y soluciones al problema de la formación, para definir estrategias de futuro.

\section{SELECCIÓN DE PARTICIPANTES}

La Subcomisión de Planificación, en la sesión celebrada el día 3 de mayo, adoptó entre otros acuerdos que la TGN se hiciera en dos sesiones, a celebrar los días 22 y 23 de mayo, seleccionándose como integrantes de las mismas a 18 Directores Generales divididos en dos grupos de nueve (5).

(5) Director de la TGN: don Angel GUILLÉn ZANON, Inspector Coordinador de la IGSAP.

Relación de asistentes a la primera sesión, 22 de mayo de 1990: don José Jorge del Castillo Pérez, Director General de Servicios del Ministerio de Obras Públicas y Urbanismo; don Javier Valero Iglesias, Director General de la IGSAP del Ministerio para las Administraciones Públicas; don Leandro González Gallardo, Director General de Personal del Ministerio de Trabajo y Seguridad Social; don José A. Sánchez Velayos, Director General de la MUFACE del Ministerio para las Administraciones Públicas; don Julián Alvarez Alvarez, Director General de Servicios del Ministerio para las Administraciones Públicas; don Manuel Bautista Pérez, Director General del Instituto Nacional de Meteorología del Ministerio de Transportes, Turismo y Comunicaciones; don Santos Fernández Castro, Director General de Servicios del Ministerio de Industria y Energía; doña Concepción Serrano Herrera, Directora del Instituto Nacional de Seguridad e Higiene en el Trabajo; don Miguel María Muñoz Medina, Director General de Tráfico del Ministerio del Interior.

Relación de asistentes a la segunda sesión, 23 de mayo de 1990: doña María Teresa Mogín Barquín, Directora General de la Función Pública del Ministerio para las Administraciones Públicas; don Luis Herrero Juan, Director de Recursos Humanos, Suministros e Instalaciones del Ministerio de Sanidad y Consumo; don José Luis Blanco Sevilla, Director General de Servicios de Ministerio de Economía y Hacienda; don Rafael Lobeto Lobo, Director General de la Marina Mercante del Ministerio de Transportes, Turismo y Comunicaciones; don Pedro de Eusebio Ribas, Director General del INEM, Ministerio de Trabajo y Seguridad Social; don José Antonio Fernández Herce, Director del Centro Español de Metrología; don Juan Pedro Serrano Arroyo, Secretario General del Fondo de Garantía Salarial del Ministerio de Trabajo y Seguridad Social; 


\section{Cuestiones Formuladas}

Las cuestiones que definitivamente se aprobaron por la Subcomisión para la celebración de la TGN respondían a los siguientes enunciados:

- Aspectos positivos de la formación del personal al servicio de la Administración pública.

- Aspectos negativos de la formación del personal al servicio de la Administración pública.

- ¿Qué se debería hacer para mejorar la formación del dicho personal?

\section{RESULTADOS OBTENIDOS}

Los resultados de una TGN dependen fundamentalmente de dos factores:

a) cuestiones que se formulan;

b) calidad del grupo de expertos seleccionados.

Teniendo en cuenta que la forma abierta de presentar las preguntas permite la aportación de soluciones creativas, la calidad de los expertos por su experiencia y conocimiento del problema constituía un factor determinante para alcanzar los objetivos propuestos. La decisión de la Subcomisión de planificación al efectuar la lista de expertos para realizar la TGN ha permitido disponer de una amplia gama de opiniones y sugerencias, que, a los efectos de su presentación, se sistematizan en tantos paneles como cuestiones se formulan, efectuándose de cada uno de ellos una valoración de los resultados obtenidos.

Por otra parte, y dado el interés que tiene para la TGN comparar los resultados obtenidos en las dos sesiones celebradas, se efectúa una comparación sistemática por paneles, para evaluar las alternativas que se producen en cada grupo y estimar el apoyo porcentual obtenido por cada una de ellas.

doña Soledad Sanz Salas, Subdirectora General de Recursos Humanos de la Dirección General de Correos y Telégrafos, Ministerio de Transportes, Turismo y Comunicaciones. 


\subsection{Evaluación y comentarios a la TGN del grupo primero}

GRUPO $10^{\circ}$

PrImer PANel: Aspectos positivos de la formación del personal al servicio de la Administración pública

\begin{tabular}{|c|c|c|}
\hline Ideas expresadas & Idea resumen & Votación \\
\hline $\begin{array}{l}\text { - Mejora la profesionalidad. } \\
\text { - Actualización de conocimientos. } \\
\text { - Cualificación de colectivos concretos. } \\
\text { - Potencia la promoción profesional. } \\
\text { - Faculta para mejorar el desempeño de algunos puestos. }\end{array}$ & $\begin{array}{l}\text { La formación posibilita la } \\
\text { profesionalización. }\end{array}$ & 20 \\
\hline $\begin{array}{l}\text { - Colaboración en la implantación y desarrollo de } \\
\text { nuevas tecnologías. } \\
\text { - Introducción de las técnicas de gestión en el ám- } \\
\text { biro de la Administración. } \\
\text { - Apertura de la Administración a técnicas y expe- } \\
\text { riencias externas. } \\
\text { - Permite la adquisición de nuevos conocimientos } \\
\text { tecnológicos. }\end{array}$ & $\begin{array}{l}\text { La formación permite la } \\
\text { incorporación de nuevas } \\
\text { Tecnologías para la ges- } \\
\text { tión. }\end{array}$ & 13 \\
\hline
\end{tabular}

- Instrumento de promoción.

- Los funcionarios de los grupos C y D la perciben

La formación fomenta la como un instrumento potencial de ascenso.

- Motiva al personal para perfeccionarse profesionalmente.

- Elementos motivador e incentivador.

- Conocimiento de los compañeros.

- Facilita la convivencia e intercambio.

La formación posibilita la convivencia e intercambio.

- Vinculación a la problemática de la gestión diaria.

- Aproximación a los modos de trabajo administrativos.

- Formación de grupos con criterios homogéneos.

La formación mejora la 2 gestión diaria.

- Facilita los procesos de adjudicación de puestos de trabajo.

La formación mejora el

- Permite una utilización más fiable de los concursos de méritos. proceso de adjudicación de los puestos de trabajo.

- Posibilita destino adecuado a vocación.

- Hay posibilidades de que las DG organicen su plan de formación propio.

- Se ajusta mejor a las necesidades internas de cada organismo cuando es organizada por dicho organismo.

\begin{tabular}{lcc}
\hline - Ha generado una fuerte demanda. & - & 1 \\
\hline - Conecta mejor con la cultura de los funcionarios. & - & 1 \\
\hline $\begin{array}{l}\text { Tiende a una formación muy general (bastante } \\
\text { polivalente). }\end{array}$ & - & 0 \\
\hline - Contactos previos con la Administración. & - & 0 \\
\hline - Universalidad de sus destinatarios. & - & 0 \\
\hline
\end{tabular}


Comentarios al contenido del primer panel (grupo 1. ${ }^{\circ}$ )

El primer panel dio lugar a 20 ideas, que se agruparon en 12 alternativas. Para proceder a priorizar el contenido de las alternativas expuestas cada miembro del grupo dispuso de siete votos.

De los 63 votos posibles únicamente se emitieron 54 y, de éstos, 44 se concentran en las tres primeras alternativas, es decir, el 81,8 por 100 de los votos emitidos, mientras que las ocho alternativas restantes reúnen el 19,2 por 100 de los votos.

Esto da lugar a una primera consecuencia:

El grupo no está muy conforme con el conjunto de ideas positivas, por lo que se reserva parte de los votos por no considerarse individualmente suficientemente identificado con las ideas surgidas.

En este primer panel, y especialmente en las tres primeras alternativas, el grupo, de acuerdo con lo manifestado en el posterior debate, refleja más bien un deseo o una estimación de lo que la formación debería representar.

El resto de las alternativas muestran aspectos positivos que suscitan un apoyo muy restringido del grupo o incluso sin ningún tipo de apoyo.

Como resumen del contenido de este primer panel podría decirse que existe un fuerte consenso del grupo sobre aspectos positivos deseables que deben producirse como consecuencia de una política correcta de formación y cuya importancia porcentual sobre el voto emitido resulta la siguiente:

\begin{tabular}{|c|c|c|}
\hline 1 & Alternativa & $\begin{array}{c}\text { Porcentaje } \\
\text { voto }\end{array}$ \\
\hline - La mejora de la pr & , & 37 \\
\hline - La incorporación & 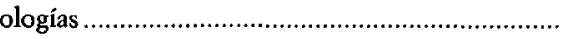 & 24 \\
\hline - La potenciación $\mathrm{d}$ & , & 20 \\
\hline
\end{tabular}

Sobre el resto de las alternativas, con apoyo más restringido, resalta el aspecto positivo de la formación en cuanto instrumento que mejora:

\begin{tabular}{|c|c|}
\hline Alternativa & $\begin{array}{c}\text { Porcentaje } \\
\text { voto }\end{array}$ \\
\hline - La convivencia entre los funcionarios & 7,0 \\
\hline - La gestión diaria del proceso burocrático & 3,7 \\
\hline - La adjudicación de puestos de trabajo & 3,7 \\
\hline
\end{tabular}


Si bien estos aspectos revelan mejoras puramente instrumentales de menor calado y contenido, la tibieza de las adhesiones a las mismas denota que el grupo las percibe como aspectos muy colaterales a los que habitualmente deben derivarse de un sistema de formación, aun cuando sean positivos como efectos derivados.

En el debate con que se cerró la TGN, el grupo mostró su clara disconformidad con los resultados de este panel, a la vista del contenido y de los resultados de los otros paneles.

\section{GRUPO $10^{\circ}$}

SEGUNDO PANEL: Aspectos negativos de la formación del personal al servicio de la Administración puiblica

\begin{tabular}{|c|c|c|}
\hline Ideas expresadas & Idea resumen & Votaciön \\
\hline $\begin{array}{l}\text { - Falta de adecuación a las realidades administra- } \\
\text { tivas. } \\
\text { - Se limita a los aspectos generales de la actividad. } \\
\text { - Desconexión entre las necesidades reales de la Ad- } \\
\text { ministración y los cursos impartidos. } \\
\text { - Falta de coordinación entre la oferta y las necesi- } \\
\text { dades de instituciones y organismos. } \\
\text { - Desvinculada de los planes de actuación de la Di- } \\
\text { rección General. } \\
\text { - No se integra en el conjunto de la organización. } \\
\text { - No adecuación a las necesidades de los diversos } \\
\text { sectores. } \\
\text { - Desconexión con las necesidades reales de la Ad. } \\
\text { ministración (o de cada organismo). }\end{array}$ & $\begin{array}{l}\text { La formación actual deno- } \\
\text { ta una falta de adecuación } \\
\text { con las necesidades reales } \\
\text { de la Administración. }\end{array}$ & 12 \\
\hline
\end{tabular}

- Escasa innovación en técnicas nuevas.

La formación no se conci-

- No incide suficientemente en nuevas tecnologías.

- No se concibe como un factor estratégico de cambio.

be como factor estratégico

de cambio.

- Ignora la importancia práctica de los idiomas.

- Falta de formación específica en el área de organización.

- No resuelve las necesidades de formación para la gestión directiva.

- Falta de planes de adecuación de la formación.

- Inexistencia de planes sistematizados de forma-

Falta un plan de formación.

- Falta de rigor en la selección del cursillista.

- Se concibe excesivamente como factor de promoción personal.

- La iniciativa de asistir a los cursos se deja a los funcionarios.

- Falta de formación orientada al puesto de trabajo por la elección libre del funcionario de los cursos a los que desea asistir.

- Puede actuar como factor desencadenante de una gran movilidad. ción. 


\begin{tabular}{|c|c|c|}
\hline Ideas expresadas & Idea resumen & Volación \\
\hline \multicolumn{3}{|l|}{$\begin{array}{l}\text { - En oferta libre acuden más quienes menos trabajo } \\
\text { tienen. } \\
\text { - Permite que haya el «profesional» del cursillo. }\end{array}$} \\
\hline $\begin{array}{l}\text { - No conecta con la carrera profesional. } \\
\text { - Desconexión entre formación y carrera adminis- } \\
\text { trativa. } \\
\text { - No se contempla como apoyo continuo a la carre- } \\
\text { ra profesional. }\end{array}$ & $\begin{array}{l}\text { Desvinculación de la carre- } \\
\text { ra profesional y el concep- } \\
\text { to de formación. }\end{array}$ & 4 \\
\hline $\begin{array}{l}\text { - Medios muy insuficientes. } \\
\text { - Falta de cobertura presupuestaria para los planes } \\
\text { de formación (insuficiencia del capítulo I del pre- } \\
\text { supuesto). }\end{array}$ & $\begin{array}{l}\text { Falta de medios para la } \\
\text { formación. }\end{array}$ & 3 \\
\hline $\begin{array}{l}\text { - Falta adecuación entre oferta y demanda. } \\
\text { - Desajuste cualitativo y cuantitativo entre oferta y } \\
\text { demanda. }\end{array}$ & $\begin{array}{l}\text { Desajuste entre oferta y de- } \\
\text { manda. }\end{array}$ & 2 \\
\hline $\begin{array}{l}\text { - Insuficiente profesionalización del profesorado. } \\
\text { - Profesorado generalmente no preparado. } \\
\text { - El profesorado no es siempre el mejor. }\end{array}$ & Profesorado deficiente. & 2 \\
\hline — No resuelve bien la combinación teoría-práctica. & - & - \\
\hline
\end{tabular}

Comentarios al contenido del segundo panel (grupo $1 .^{\circ}$ )

En los aspectos negativos el grupo produce 25 ideas que se agrupan en ocho alternativas, para cuya priorización dispone de cinco votos cada uno.

Se emiten los 45 votos posibles y de ellos el 61 por 100 se concentran en las tres primeras alternativas con el siguiente reparto porcentual:

\begin{tabular}{|c|c|}
\hline Alternativa & $\begin{array}{c}\text { Porcentaje } \\
\text { voto }\end{array}$ \\
\hline $\begin{array}{l}\text { - Falta de adecuación a las necesidades reales de la Administración } \\
\text { - No se concibe como un factor estratégico de cambio } \\
\text { - La falta de un plan de formación }\end{array}$ & $\begin{array}{l}26 \\
17 \\
17\end{array}$ \\
\hline
\end{tabular}

Se trata de tres posiciones muy sólidas dentro del grupo, y se señalan como las carencias más importantes del sistema de formación, carencias que pudieran sintetizarse en la necesidad de adecuar cursos y tecnologías a las exigencias de los distintos Centros Directivos y al proceso de cambio en la Administración de forma planificada. 
Es decir, se señala como muy negativo para la organización que los cursos no se hagan pensando en la misma y que la formación no sea considerada como factor estratégico para la modernización y el cambio.

Esta desvinculación convierte a los cursos de formación, a juicio del grupo, en una «fábrica» de títulos o certificados cuya utilidad principal es la acumulación de «puntos» para ulteriores concursos de méritos, concentrando esta opinión el 16 por 100 de los votos emitidos.

Por lo que respecta al resto de los votos, éstos se distribuyen entre las cuatro alternativas restantes de las siguientes manera:

\begin{tabular}{|c|c|}
\hline Allernativa & $\begin{array}{c}\text { Porcentaje } \\
\text { voto }\end{array}$ \\
\hline - Desvinculación entre la carrera profesional y el proceso de formación................... & 8 \\
\hline 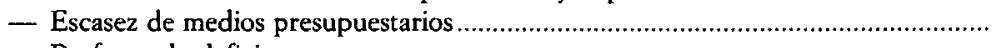 & 7 \\
\hline - Profesorado deficiente & 4 \\
\hline 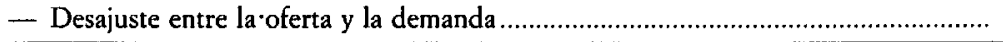 & 4 \\
\hline
\end{tabular}

\section{GRUPO 1.}

TERCER PANEL: Mejoras a introducir en la formación del personal al servicio de la Administración pública

\begin{tabular}{llc}
\hline \multicolumn{1}{c}{ Ideas expresadas } & \multicolumn{1}{c}{ Idea resumen } & Votación \\
\hline - Ayudar a crear o desarrollar centros de formación & $\begin{array}{l}\text { Debe potenciar la forma- } \\
\text { en los propios organismos. } \\
\text { los Centrontralizada postores. }\end{array}$ \\
- Elaboración de planes adecuados. & \\
- Estructurar dos niveles: & \\
- El general por una institución común. & \\
- El específico por órganos gestores. & \\
- Potenciar las unidades de formación en los De- & \\
- Trantamentos. & \\
tre el INAP y y los Departamentos. & \\
- Desconcentración de planes de formación sectoria- & \\
les (bien dotados). & \\
\hline - Diseñar los cursos en función de las necesidades & La formación debe adap- \\
de las Direcciones Generales. & tarse a las necesidades de \\
- Estudio conjunto con órganos gestores para defi- & los Centros Gestores. \\
nir planes de formación. & \\
- Estructurar la formación de acuerdo con las nece- \\
sidades de los organismos. \\
- Dirigir la formación a las necesidades de las or- \\
ganizaciones. \\
- Los cursos deben programarse atendiendo las pe- \\
ticiones de los Centros Gestores. \\
- Contar con las instituciones y centros en la elabo- \\
ración del plan. \\
Deberá dar respuesta a las necesidades prácticas de \\
las distintas organizaciones. \\
\hline
\end{tabular}




\begin{tabular}{|c|c|c|}
\hline Ideas expresadas & Idea resumen & Votación \\
\hline $\begin{array}{l}\text { - Diseñar una política de formación específica para } \\
\text { Directivos. } \\
\text { - Mecanismo para la innovación de técnicas de Di- } \\
\text { rección y Gestión. } \\
\text { - Especial hincapié en formación de Directivos. Téc- } \\
\text { nica de Management. }\end{array}$ & $\begin{array}{l}\text { Debe posibilitar la forma- } \\
\text { ción de Directivos. }\end{array}$ & 6 \\
\hline
\end{tabular}

- Máxima atención a las nuevas tecnologías.

Debe fomentar las nuevas

- Importancia de idiomas (técnicas avanzadas). tecnologías y la innovación.

- Especial atención a los aspectos más innovados.

- Que los cursos sean innovadores en técnicas no reproductoras de lo antiguo.

- Que sean las Direcciones Generales las que decidan quiénes deben asistir a los cursos.

Debe mejorar el sistema de $\quad 6$

- Deben establecerse sistemas objetivos de selección selección de alumnos. de alumnos.

- Elegir alumnado.

- Crear nueva cultura.

Debe posibilitar el avanzar

- Elaborar un catálogo de «Ideas Fuerza» que sustenten y orienten el plan de formación.

- Medios.

hacia la nueva cultura.

6

- Aumentar sustancialmente los medios que se destinen a la formación.

Deben aumentarse los me-

dios para la formación.

- Deben ser un mecanismo de mejora de los conocimientos profesionales.

- Deberá mejorar la adecuación persona/puesto.

- Diseñar planes de formación para poder cubrir las vacantes con buenos técnicos.

Debe proporcionar mayor

- Conectar la formación con las necesidades de los puestos de trabajo.

- Tener en consideración en la elaboración de los programas el principio de mejora de la gestión y el carácter práctico que debe darse al curso.

- Diseñar los programas orientados al ejercicio profesional y al puesto de trabajo.

- Semiprofesionalizar al profesorado.

- Deben hacerse acciones de formación del profesorado.

profesionalidad.

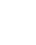

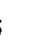

- Seleccionar y capacitar adecuadamente al profesorado. Deben seleccionar mejor al 2
profesorado.

— Valoración sólo de ciertos cursos. 
Comentarios al contenido del tercer panel (grupo 1..$\left.^{\circ}\right)$

El tercer panel sobre mejoras a introducir en la formación produjo nueve alternativas que agrupaban 37 ideas, disponiendo cada miembro del grupo de seis votos para jerarquizar las alternativas resultantes.

Se emiten 54 votos posibles, cuya distribución se efectuó de la siguiente manera:

\begin{tabular}{|c|c|}
\hline Allernativa & $\begin{array}{c}\text { Porcentaje } \\
\text { voto }\end{array}$ \\
\hline - Potenciación de un sistema de formación descentralizado por Centros Gestores . & 18,0 \\
\hline 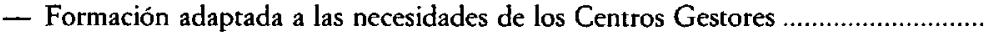 & 18,0 \\
\hline — Formación de Directivos & 11,5 \\
\hline - Fomento de las nuevas tecnologías & 11,5 \\
\hline 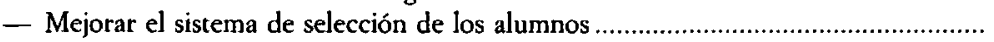 & 11,5 \\
\hline 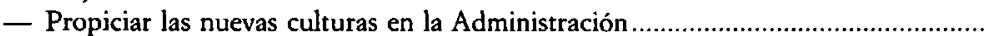 & 9,5 \\
\hline - Incremento de los medios presupuestarios & 9,5 \\
\hline — Mejora de la profesionalización & 7,5 \\
\hline - Selección del profesorado & 3,5 \\
\hline
\end{tabular}

Del conjunto de esta distribución se percibe en el grupo como primera mejora a introducir la potenciación de los centros de formación departamentales para que sean los propios Centros Gestores quienes aborden los planes sectoriales de formación; se sugiere la distinción entre dos niveles de formación: el general, que debería ser abordado por el INAP, y los sectoriales o específicos, dirigidos a proporcionar el conocimiento de técnicas específicas a colectivos determinados, en función de las necesidades de cada centro, y que deberían abordar los propios Centros Gestores.

Con la misma relevancia, el grupo apoya la necesidad de adaptar la formación a las necesidades específicas de los centros gestores, lo que exige que los planes de formación se elaboren en coordinación con los centros directivos departamentales.

Junto a estas dos alternativas de mejora, que reciben un apoyo similar del grupo, aparecen tres alternativas a las que el grupo otorga idéntica relevancia, como son:

- La necesidad de reforzar la formación de directivos.

- El fomento de las nuevas tecnologías.

- La revisión del sistema de selección de alumnos.

Todas ellas dirigidas a potenciar el proceso de modernización en la Administración como una clara apuesta por la implantación de un sistema de formación que permita introducir las técnicas gerenciales concibiendo los cursos de formación como una herramienta de trans- 
formación hacia la nueva cultura administrativa y como estrategia imprescindible para abordar el proceso de cambio.

Las restantes alternativas suponen un conjunto de sugerencias coherentes con los cambios de orientación antes mencionados, como son:

- El aumento de los medios presupuestarios.

- La formación como vehículo para permitir una mayor profesionalización.

— La mejora del profesorado.

\subsection{Evaluación y comentarios a la TGN del grupo segundo}

\section{GRUPO 2.}

PRIMER PANEL: Aspectos positivos de la formación del personal al servicio de la Administración pública

\begin{tabular}{llc}
\hline \multicolumn{1}{c}{ Ideas expresadas } & \multicolumn{1}{c}{ Idea resumen } & Votación \\
\hline - Incipiente sensibilidad por el tema. & $\begin{array}{l}\text { Amplio consenso sobre la } \\
\text { necesidad de mejorar la }\end{array}$ & 12 \\
- Existencia de un amplio consenso sobre la necesi- & \\
dad de potenciar y mejorar la formación. & formación. & \\
- Coincidencia sobre la necesidad de ordenar un sis- & & \\
tema global y flexible. & & \\
- Creciente interés de la Administración y los servi- \\
dores públicos por la formación.
\end{tabular}

- Nivel de entrada para los grupos A.

- Formación como parte del proceso selectivo.

- Formación inmediata al acceso a la función pública, mantenimiento e identificación de las causas ac-

La formación mejora. Setuales como parte del proceso de selección.

- Algunas de sus actividades dirigidas a responder a lección-formación en el grupo A. necesidades específicas de colectivos concretos.

- La formación jurídico-administrativa.

- Los cursos sectoriales y de especialización.

- El contenido de ciertos cursos.

- El sector sanitario parece muy apropiado y aceptado el sistema de formación de los MIR.

- Formación para participar en distintas áreas de actividad en la Función Pública.

cursos es positivos.

- Formación para tareas específicas de Dirección. 


\begin{tabular}{llc}
\hline \multicolumn{1}{c}{ Ideas expresadas } & \multicolumn{1}{c}{ Idea resumen } & Votación \\
\hline - La gestión centralizada en el INAP. & $\begin{array}{l}\text { El INAP como Institución } \\
\text { prospectiva de la forma- } \\
\text { - Su concepción como Escuela Nacional que debe te- } \\
\begin{array}{l}\text { ner una visión de conjunto de las necesidades de } \\
\text { formación. }\end{array}\end{array}$ & 6 \\
\hline - Colaboración del personal de la Administración & 3 \\
\hline como docentes. & 2 \\
\hline Participación del personal periférico. & 2 \\
\hline $\begin{array}{l}\text { Dotaciones significativas para formación a nivel glo- } \\
\text { bal }\end{array}$ & 1 \\
\hline $\begin{array}{l}\text { Existencia de una infraestructura importante: las } \\
\text { diversas escuelas de formación. }\end{array}$ & 2 \\
\hline
\end{tabular}

\section{Comentarios al contenido del primer panel (grupo 2.")}

El primer panel dio lugar a 22 ideas que se agruparon en ocho alternativas en función del contenido de las mismas. Para priorizar el conjunto de estas alternativas cada miembro del grupo dispuso de cinco votos, emitiéndose en este primer panel 41 votos (es decir, uno más de los posibles, por error de los participantes). En términos globales puede decirse que las tres primeras alternativas del primer panel suponen el 65,5 por 100 de los votos emitidos, mientras que las cinco restantes alternativas suman el 34,5 por 100 de las mismas.

Esto evidencia un consenso muy fuerte del grupo en las tres primeras, mientras que en el resto de las alternativas que surgen por agrupación de las distintas. ideas se sufre una dispersión de voto por ser ideas poco significativas en el conjunto de las expuestas.

La distribución porcentual de los votos obtenidos por cada una de las alternativas formuladas es la siguiente:

\begin{tabular}{|c|c|}
\hline Alternativa & $\begin{array}{c}\text { Porcentaje } \\
\text { volo }\end{array}$ \\
\hline 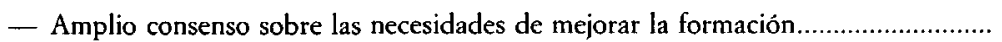 & 29,0 \\
\hline — La formación como mejora de selección del grupo A & 19,5 \\
\hline 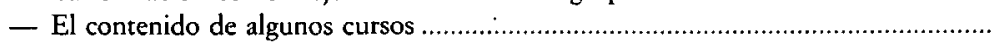 & 17,0 \\
\hline 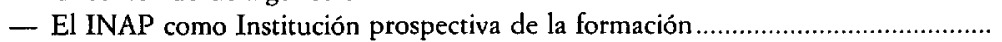 & 14,6 \\
\hline 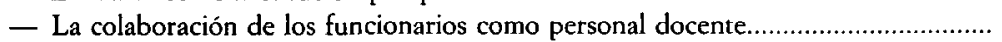 & 7,3 \\
\hline 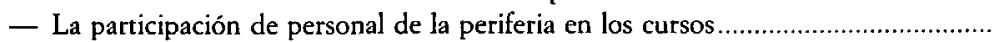 & 4,8 \\
\hline - Existencia de dotaciones significativas para la dotación a nivel global.................... & 4,8 \\
\hline - Existencia de una infraestructura importante en las diversas Escuelas de For- & 2,4 \\
\hline
\end{tabular}

Globalmente puede decirse que el grupo de expertos ve como positiva la existencia de un consenso generalizado sobre su necesidad, así como la aportación que hacen las Escuelas de Formación al proceso selectivo y de formación de los funcionarios del grupo A. 
Asimismo, el grupo estima como una situación altamente positiva el contenido de algunos cursos, como pueden ser los que versan sobre materias jurídicas o de técnicas gerenciales, sin que las otras ideas desciendan a aspectos más concretos de los cursos, que actualmente son considerados como idóneos, limitándose a hacer un enunciado bastante difuso de estos aspectos positivos.

Por otra parte, el grupo de expertos integrado en el segundo grupo considera buena la gestión centralizada del INAP, siempre y cuando actúe como una Escuela Nacional que debe tener una visión de conjunto de las necesidades de formación.

\section{GRUPO 2.}

SEGUNDO PANEL: Aspectos negativos de la formación del personal al servicio de la Administración pública

\begin{tabular}{|c|c|c|}
\hline Ideas expresadas & Idea resumen & Votación \\
\hline $\begin{array}{l}\text { - Cursos genéricos que se imparten por hacer algo, } \\
\text { pero no por necesidad de la Administración. } \\
\text { - Falta planificación de la formación: no está coor- } \\
\text { dinada con las necesidades. } \\
\text { - Falta de plan para las AAPP debidamente es- } \\
\text { tructurado. } \\
\text { - La selección de participantes no responde a las ne- } \\
\text { cesidades de la Administración. } \\
\text { - Ausencia de adecuada relación entre la ordenación } \\
\text { e integración del sistema y el papel periférico. } \\
\text { - El plan de formación se hace sin contar con las ne- } \\
\text { cesidades de los Centros Directivos. } \\
\text { - Falta de coordinación en la elaboración de los sis- } \\
\text { temas de formación. } \\
\text { - Desconocimiento de medios globales disponibles. } \\
\text { - Ausencia de un modelo global de formación. }\end{array}$ & $\begin{array}{l}\text { Falta de planificación y } \\
\text { coordinación con las nece- } \\
\text { sidades de la Administra- } \\
\text { ción. }\end{array}$ & 14 \\
\hline
\end{tabular}

- La información y coordinación entre el INAP y los centros directivos es escasa o inexistente.

- Falta de participación en la elaboración del contenido de los cursos de formación.

ción.

- El limitado análisis de las necesidades reales del alumnado.

- Falta de conocimiento real de las necesidades.

- Selección del personal que asiste.

- Falta de consideración de la formación como una variable estratégica para la modernización.

Contenido poco técnico de los cursos.

- Ausencia de conciencia de europeidad y modernidad.

- Inmovilidad, rigidez y lentitud en la evolución del sistema.

- Carencia de formadores y técnicos para formar a directivos.

Carencia de formadores $y$

- Falta de metodología en la definición de las técnicas, contenidos y evaluación de los cursos. de metodología para la definición y evaluación de cursos. 


\begin{tabular}{|c|c|c|}
\hline Ideas expresadas & Idea resumen & Votación \\
\hline $\begin{array}{l}\text { - Se concibe como un sistema de acumular puntos } \\
\text { para los concursos de méritos. } \\
\text { - El actual sistema fomenta la formación de cara al } \\
\text { curriculum más que a satisfacer las necesidades de } \\
\text { la organización. }\end{array}$ & $\begin{array}{l}\text { Los cursos se perciben } \\
\text { como fábrica de méritos. }\end{array}$ & 3 \\
\hline $\begin{array}{l}\text { - Necesidad de un papel más activo por parte del } \\
\text { INAP. } \\
\text { - Limitada capacidad de respuesta del INAP. } \\
\text { - Dispersión y escasa coordinación del INAP sobre } \\
\text { las demás Instituciones formadoras. }\end{array}$ & $\begin{array}{l}\text { Actuación insuficiente del } \\
\text { INAP. }\end{array}$ & 2 \\
\hline - Concepción excesivamente teórica de la formación. & $\begin{array}{l}\text { Formación excesivamente } \\
\text { teórica. }\end{array}$ & 1 \\
\hline - Falta de divulgación que fomente el interés. & 一 & - \\
\hline $\begin{array}{l}\text { - Falta de relación entre formación, carrera adminis- } \\
\text { trativa y promoción profesional. }\end{array}$ & - & - \\
\hline
\end{tabular}

\section{Comentarios al contenido del segundo panel (grupo 2.")}

Este segundo panel contiene 27 ideas o aspectos negativos del sistema de formación que posteriormente, por decisión del grupo, se integran en nueve alternativas. Para priorizar el contenido de los mismas se dispone de cinco votos por cada miembro del grupo, emitiéndose 38 sobre los 40 votos posibles. Resaltando. la importancia que se otorga al contenido de las dos primeras alternativas, cuya suma global constituye el 63,1 por 100 de los votos emitidos, distribuyéndose el 36,9 por 100 de los votos restantes entre las otras siete alternativas.

La distribución porcentual de los votos emitidos entre el conjunto de las nueve alternativas son:

\begin{tabular}{|c|c|}
\hline Alternativa & $\begin{array}{c}\text { Porcentaje } \\
\text { voto }\end{array}$ \\
\hline Falta de planificación y coordinación con las necesidades de la Administración... & 36,8 \\
\hline 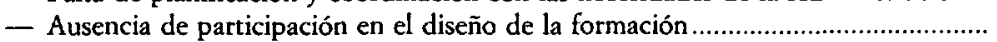 & 26,3 \\
\hline 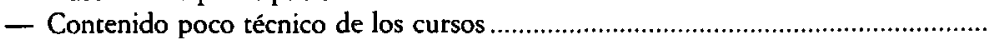 & 10,5 \\
\hline - Carencia de formadores y metodologías para la definición y evaluación de los & \\
\hline cursos & 10,5 \\
\hline 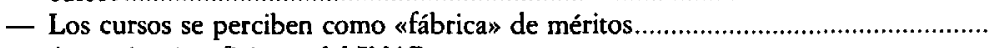 & 7,8 \\
\hline - Actuación insuficiente del INAP. & 5,2 \\
\hline 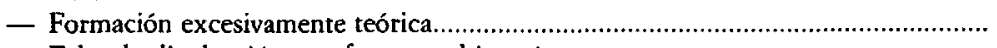 & 2,6 \\
\hline 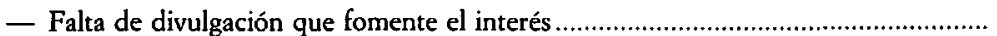 & 0,0 \\
\hline 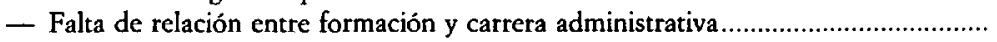 & 0,0 \\
\hline
\end{tabular}

Los comentarios que suscitan estos datos revelan que el grupo detecta como muy negativo para el proceso actual de formación tanto la 
falta de planificación y coordinación con las necesidades de la Administración como la ausencia de participación de los Centros Directivos en el diseño de las mismas. El grupo tuvo muchas dificultades para distinguir entre estas dos alternativas, toda vez que de una lectura atenta del contenido de las ideas expresadas en cada una de ellas pudiera decirse que constituían una única alternativa, ya que la ausencia de participación en el diseño de la formación es una de las causas de que no exista conexión real entre la actual formación y las necesidades de la Administración, lo que imposibilita planificar un sistema de formación que responda a las exigencias de modernización de la Administración.

Esta falta de acuerdo del grupo para unificar ambas alternativas aconsejó mantenerlas como distintas toda vez que de acuerdo con la TGN, de no existir una unanimidad absoluta para su unificación, deben mantenerse todas las alternativas expuestas por separado para que el grupo decida posteriormente con las votaciones.

El resto de las alternativas revelan aspectos negativos, como el contenido poco técnico de los cursos, o la idea que pone de manifiesto que la formación actual no supone una estrategia decisiva para la modernización de las administraciones. Por otra parte, otra alternativa hace referencia a la carencia de formadores y de metodología en el proceso de formación, para terminar manifiestando que el INAP, con un papel insuficiente, está actuando como una fábrica de emitir títulos que además se sustentan en una formación excesivamente teórica y poco práctica.

\section{GRUPO 2."}

TERCER PANEL: Mejoras a introducir en la formación del personal al servicio de la Administración pública

\begin{tabular}{llc}
\hline \multicolumn{1}{c}{ Ideas expresadas } & \multicolumn{1}{c}{ Idea resumen } & Votación \\
\hline - Establecer un sistema de detección de necesidades. & $\begin{array}{l}\text { Deben detectarse las nece- } \\
\text { sidades previamente. }\end{array}$ & 9 \\
- Determinar áreas concretas de formación. & \\
- Definir las necesidades en materia de formación de & & \\
Directivos, Gestores Técnicos y restante personal. & & \\
\hline - Prioridad a la formación de Directivos. & $\begin{array}{l}\text { Debe acometerse la forma- } \\
\text { ción de Directivos. }\end{array}$ \\
- Formación del personal Directivo. & \\
- Objetivos prioritarios de la formación (grupo A): & \\
- Directivos. & \\
- Técnicas Management. & \\
- Recursos humanos. & \\
- Funcionarios entrada. & \\
Formación especializada (Directivos, Gerencia, In- \\
formática, RH, laboral, impuestos, etc.) que posi- \\
bilite además la promoción y la carrera admi- \\
nistrativa.
\end{tabular}




\begin{tabular}{llc}
\hline \multicolumn{1}{c}{ Ideas expresadas } & \multicolumn{1}{c}{ Idea resumen } & Votación \\
\hline - Formación a la umedidas de las necesidades de los & $\begin{array}{l}\text { Formación versus necesida- } \\
\text { Centros Directivos. }\end{array}$ & 6 \\
\hline - Mejora de la relación entre motivación y for- & $\begin{array}{l}\text { Reforzar la motivación y la } \\
\text { mación. }\end{array}$ & 6 \\
- Información. \\
pación.
\end{tabular}

\section{Comentarios al contenido del tercer panel (grupo 2.")}

El tercer panel, sobre mejoras a introducir en el sistema de formación, produjo 24 ideas que se integraron en 10 alternativas, para cuya priorización el grupo dispuso de siete votos. Se emitieron todos los posibles, es decir, 49, al haberse ausentado en la formación de este último panel uno de los miembros del grupo.

Respecto a la distribución porcentual de los votos emitidos por cada una de las alternativas cabe decir que hay un gran equilibrio entre el conjunto de los votos emitidos, moviéndose los porcentajes entre el 4 
y 12 por 100 , salvo en la primera alternativa, que recibe un 18,3 por 100 , quedando como sigue:

\begin{tabular}{|c|c|}
\hline Alternativa & $\begin{array}{c}\text { Porcentaje } \\
\text { voto }\end{array}$ \\
\hline - Deben detectarse las necesidades públicamente. & 18,3 \\
\hline - Debe acometerse la formación de directivos ........ & 12,2 \\
\hline - La formación como respuesta a las necesidades de la Administración...................... & 12,2 \\
\hline 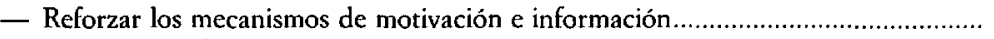 & 12,2 \\
\hline - Equilibrar la formación centralizada y descentralizada & 10,2 \\
\hline 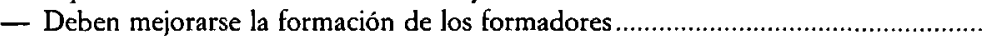 & 10,2 \\
\hline — Implantación de técnicas de evaluación. & 8,0 \\
\hline - El INAP como motor de cambio del proceso formativo ............................. & 8,0 \\
\hline - Diseñar la formación como un proceso continuado. & 4,3 \\
\hline - Establecimiento de nexos de conexión con la formación europea ................................. & 4,3 \\
\hline
\end{tabular}

Del conjunto de las ideas expuestas y de las alternativas en las que se agrupan las mismas destaca la necesidad de establecer un sistema de detección de necesidades como paso previo a poder establecer una planificación en la formación. Resalta asimismo la prioridad en acometer la formación de directivos mediante la impartición de técnicas gerenciales que permitan formar auténticos gerentes públicos que conduzcan el proceso de modernización.

Del resto de las ideas manifestadas merece destacarse por su incidencia en el proceso de formación el papel que se pide que asuma el INAP como impulsor y coordinador de toda la política a nivel de Estado, diseñando cursos donde se impartan técnicas y se evalúen sus resultados, se acometa un proceso de mejora de los formadores, como elementos indispensables del proceso de formación. En este nuevo papel que debe asumir el INAP debe darse un equilibrio entre la dirección centralizada del proceso de formación y la ejecución descentralizada de aspectos sectoriales de la misma. 
5.3. Examen comparado de los resultados obtenidos

PRIMER PANel: Aspectos positivos de la formación del personal al servicio de la Administración pública

\begin{tabular}{|c|c|c|c|c|c|c|}
\hline & $\begin{array}{l}\text { Grupo primero } \\
\text { (Sesión de } 22 \text { de mayo de 1990) }\end{array}$ & $\begin{array}{l}\text { Voto } D(*) \text { : } \\
\text { Voto } E(* *):\end{array}$ & $\begin{array}{l}63 \\
55\end{array}$ & $\begin{array}{l}\text { Grupo segundo } \\
\text { (Sesión de } 23 \text { de mayo de 1990) }\end{array}$ & $\begin{array}{l}\text { Voto } D(*) \text { : } \\
\text { Voto } E(* *) \text { : }\end{array}$ & $\begin{array}{l}40 \\
41\end{array}$ \\
\hline & Alternativas resultantes & Votación & $\%$ sobre voto $E$ & Alternativas resultantes & Volación & $\%$ sabre voto $E$ \\
\hline 2." & Permite incorporar nuevas tecnologias & 13 & 24,0 & $\begin{array}{l}\text { 2." Mejora la selección de funcionarios del gru- } \\
\text { po «A» }\end{array}$ & 8 & 19,5 \\
\hline 3." & Fomenta la motivación & 11 & 20,0 & 3. El contenido de algunos cursos & 7 & 17,0 . \\
\hline 6." ${ }^{2}$ & $\begin{array}{l}\text { Mejora del proceso de adjudicación de puestos } \\
\text { Mejora la formación cuando la organiza cada }\end{array}$ & 2 & 3,7 & 6. Participación del personal periférico & 2 & 4,8 \\
\hline & Centro Gestor & 1 & 1,8 & $\begin{array}{l}\text { 7.2 Dotaciones significativas a nivel global para } \\
\text { formación }\end{array}$ & 2 & 4,8 \\
\hline 8." & Ha generado una fuerte demanda & 1 & 1,8 & $\begin{array}{l}\text { 8." Existencia de infraestructura importante: Es- } \\
\text { cuela de formación }\end{array}$ & 1 & 2,4 \\
\hline
\end{tabular}

(\#) Voto D: Voto disponible.

(*) Voro E: emitido. 
SEGUNDO PANEL: Aspectos negativos de la formación del personal al servicio de la Administración pública

\begin{tabular}{|c|c|c|c|c|c|c|c|}
\hline & $\begin{array}{l}\text { Grupo primero } \\
\text { (Sesión de } 22 \text { de mayo de 1990) }\end{array}$ & $\begin{array}{l}\text { Voto } \mathrm{D}\left({ }^{*}\right): \\
\text { Voto } E\left({ }^{(*)}\right) \text { : }\end{array}$ & $\begin{array}{l}45 \\
45\end{array}$ & & $\begin{array}{l}\text { Grupo segundo } \\
\text { (Sesión de } 23 \text { de mayo de 1990) }\end{array}$ & $\begin{array}{l}\text { Voto } \mathrm{D}\left({ }^{*}\right): \\
\text { Voto } \mathrm{E}\left({ }^{* *}\right):\end{array}$ & $\begin{array}{l}40 \\
38\end{array}$ \\
\hline & Aliernativas resultantes & Votación & \% sobre voto $E$ & & Alternativas resultantes & Votación & $\%$ sobre voto $E$ \\
\hline $1 . *$ & $\begin{array}{l}\text { Falta de adecuación con las necesidades reales } \\
\text { de la Administración }\end{array}$ & 12 & 26,0 & 1." & $\begin{array}{l}\text { Falta de planificación y coordinación con las ne- } \\
\text { cesidades de la Administración }\end{array}$ & 14 & 36,8 \\
\hline $2 .^{a}$ & $\begin{array}{l}\text { No se concibe como factor estratégico del } \\
\text { cambio }\end{array}$ & 8 & 17,0 & $2 .^{*}$ & $\begin{array}{l}\text { Ausencia de participación en el diseño de la } \\
\text { formación }\end{array}$ & 10 & 26,3 \\
\hline 3." & Falta un plan de formación & 8 & 17,0 & $3.0^{\circ}$ & Contenido poco técnico de los cursos & 4 & 10,5 \\
\hline $4 .^{*}$ & Falta de rigor en la selección del cursillista & 7 & 16,0 & 4." & Carencia de formadores y metodologia & 4 & 10,5 \\
\hline 5." & $\begin{array}{l}\text { Desvinculación entre cartera profesional y for- } \\
\text { mación }\end{array}$ & 4 & 8,0 & $5 .{ }^{a}$ & $\begin{array}{l}\text { Los cursos se perciben como una «fábrica de } \\
\text { méritos» }\end{array}$ & 3 & 7,8 \\
\hline \multirow{4}{*}{$\begin{array}{l}\text { 6." } \\
\text { 7." } \\
8 .^{a} \\
9 .\end{array}$} & Falta de medios para la formación & 3 & 7,0 & $6 .{ }^{\circ}$ & Actuación insuficiente del INAP & 2 & 5,2 \\
\hline & Desajustes entre oferta y demanda & 2 & 4,4 & $7 .{ }^{\circ}$ & Formación excesivamente teórica & 1 & 2,6 \\
\hline & $\begin{array}{l}\text { Profesorado deficiente } \\
\text { No resuelve bien la combinación entre teoría y }\end{array}$ & 2 & 4,4 & $8 .^{n}$ & Falta de divulgación que fomente el interés & - & - \\
\hline & práctica & - & - & $9 .{ }^{\circ}$ & $\begin{array}{l}\text { Falta relación entre formación y carrera ad- } \\
\text { ministrativa }\end{array}$ & - & - \\
\hline
\end{tabular}

(*) Voto D: Voto disponible.

(*) Voto E: emitido. 
TERCER PANEL: Mejoras a introducir en la formación del Personal al Servicio de la Administración Pública

\begin{tabular}{|c|c|c|c|c|c|c|c|}
\hline & $\begin{array}{l}\text { Grupo primero } \\
\text { (Sesión de } 22 \text { de mayo de 1990) }\end{array}$ & $\begin{array}{l}\text { Voto D }\left(^{*}\right): \\
\text { Voto } \mathrm{E}(* *):\end{array}$ & $\begin{array}{l}54 \\
54\end{array}$ & & $\begin{array}{l}\text { Grupo segundo } \\
\text { (Sesión de } 23 \text { de mayo de 1990) }\end{array}$ & $\begin{array}{l}\text { Voto } \mathrm{D}\left({ }^{*}\right): \\
\text { Voto } \mathrm{E}( \pm) \text { : }\end{array}$ & $\begin{array}{l}49 \\
49\end{array}$ \\
\hline & Alternativas resultantes & Votación & $\%$ sobre voto $E$ & & Alternativas resultantes & Votación & $\%$ sobre voto $E$ \\
\hline 1." & $\begin{array}{l}\text { Potenciar la formación a través de los Centros } \\
\text { Gestores }\end{array}$ & 10 & 18,0 & 1." & $\begin{array}{l}\text { Necesidad de detectar previamente las ne- } \\
\text { cesidades }\end{array}$ & 9 & 18,3 \\
\hline 2:" & $\begin{array}{l}\text { La formación debe adaptarse a las necesidades } \\
\text { de los Centros Gestores }\end{array}$ & 10 & 18,0 & $2{ }^{a}$ & Debe acometerse la formación de directivos & 6 & 12,2 \\
\hline 3." & $\begin{array}{l}\text { Debe posibilitar la formación de directivos } \\
\text { Debe fomentar las nuevas tecnologias y la in- }\end{array}$ & 6 & 11,5 & $3 .^{\mathrm{a}}$ & Formación como respuesta a las necesidades & 6 & 12,2 \\
\hline 5.* & $\begin{array}{l}\text { novación } \\
\text { Debe mejorar el sistema de selección de alum. }\end{array}$ & 6 & 11,5 & $4 .^{\prime \prime}$ & Reforzar la motivación y la información & 6 & 12,2 \\
\hline & nos & 6 & 11,5 & 5." & $\begin{array}{l}\text { Equilibar la formación centralizada y des- } \\
\text { centralizada }\end{array}$ & 5 & 10,2 \\
\hline 6." & Debe posibilitar el avance hacia la nueva cultura & 5 & 9,5 & 6. & Prioridad a la formación de los formadores & 5 & 10,2 \\
\hline 7." & Deben aumentarse los medios para la formación & 5 & 9,5 & 7.a & Implantar técnicas de evaluación & 4 & 8,0 \\
\hline 8." & Debe proporcionar mayor profesionalidad & 4 & 7,5 & 8. & El INAP debe asumir un nuevo papel & 4 & 8,0 \\
\hline 9." & Debe seleccionar mejor el profesorado & 2 & 3,5 & $\begin{aligned} 9.9^{\circ} \\
10.0^{\circ}\end{aligned}$ & $\begin{array}{l}\text { Debe tenderse a la formación continuada } \\
\text { Conexión de la formación con los sistemas } \\
\text { europeos }\end{array}$ & $\begin{array}{l}1 \\
1\end{array}$ & $\begin{array}{l}2,0 \\
2,0\end{array}$ \\
\hline
\end{tabular}




\section{Comentarios}

Como consecuencia del examen comparado de los resultados obtenidos por los dos grupos de expertos en la elaboración de los tres paneles de la TGN, aparecen los siguientes resultados:

\section{Primer panel}

Existe una clara divergencia entre el primer grupo y el segundo. Como ya se comentó, en este panel el primer grupo se fijó más en los aspectos deseables que debieran existir en un sistema de formación antes que en poner de manifiesto los aspectos positivos que se están produciendo en el sistema actual.

Por ello, mientras que en el segundo grupo se fijan en aspectos más concretos haciendo referencia a temas como los efectos de la formación en la selección actual de funcionarios del grupo A, el contenido de algunos cursos, la participación del personal periférico, el papel del INAP, etc., en el grupo primero se resaltan aspectos poco concretos y cuando coinciden en alguno de ellos son claramente discrepantes, como sucede en el papel que juega el INAP en el sistema de formación.

Podemos decir que mientras el grupo primero se ha fijado en lo deseable, el segundo grupo se ha fijado en lo positivo del sistema actual y por ello quizá se han encontrado menos aspectos positivos en el grupo segundo que en el grupo primero.

No obstante, es preciso resaltar la dificultad que siempre conlleva la configuración de este tipo de panel, pues se trata de analizar una situación que por definición no responde a las exigencias de eficacia y eficiencia y en donde, lógicamente, los aspectos positivos son difíciles de identificar.

\section{Segundo panel}

El segundo panel, sobre aspectos negativos de la formación, recoge el mismo número de alternativas en cada grupo, si bien la última alternativa del primer grupo y las dos últimas del segundo grupo no reciben ningún voto. Esto supone que en este último grupo se concentran los votos en las siete alternativas primeras, recibiendo la primera de ellas, relativa a «la falta de planificación y coordinación con las necesidades de la Administración» el 36,8 por 100 de los votos emitidos, es decir, un 10 por 100 más que la alternativa más votada en el grupo primero y que se refiere al mismo tema.

En cualquier caso, hay que resaltar que las dos primeras alternativas de ambos grupos se refieren al «divorcio» existente entre forma- 
ción y necesidades a cubrir, globalizando el 34,4 por 100 de los votos emitidos.

Partiendo de esta coincidencia fundamental, conviene destacar también cómo ambos grupos son sensibles a:

- La ausencia de un plan de formación.

- El valor instrumentalista de los concursos (méritos).

- El profesorado deficiente.

- La formación excesivamente teórica.

- La ausencia de vinculación entre formación y carrera profesional, etcétera.

Todos ellos aspectos negativos, que a juicio de los grupos de expertos definen o condicionan el actual panorama del sistema formativo.

\section{Tercer panel}

El último panel recoge las mejoras a introducir y aquí es preciso resaltar el consenso de ambos grupos en tres aspectos fundamentales:

1. . La formación debe instrumentarse como una respuesta a las necesidades de los centros directivos.

2. $\quad$ El papel que deben jugar los centros gestores en el proceso de formación.

3. La necesidad de acometer la formación de directivos.

Estos tres aspectos configuran las tres primeras alternativas de ambos grupos, con un apoyo similar en cuanto a porcentaje de votos emitidos, lo que viene a mostrar la coincidencia de los expertos y la exigencia de dar respuesta a estos problemas.

Asimismo existe coincidencia en la necesidad de mejorar el profesorado, reforzando la formación de formadores.

Por otra parte, hay «ideas fuerza», que aun cuando no aparecen en los dos grupos, es necesario resaltar:

- El fomento de las nuevas tecnologías en la formación.

- La implantación de técnicas de evaluación de los cursos emitidos.

- El papel coordinador que debe asumir el INAP.

- La formación como mejora de la profesionalidad, etcétera.

\section{CONCLUSIONES}

Como síntesis de los resultados obtenidos por la TGN en las dos sesiones celebradas con los grupos de expertos seleccionados cabe formular las siguientes conclusiones: 


\section{Primera: Amplio consenso}

Existe un amplio consenso sobre las carencias y deficiencias del sistema actual de formación, así como una creciente sensibilización sobre la urgencia de contar con un plan que lo mejore.

\section{Segunda: Papel del INAP}

El INAP debe asumir un papel coordinador de la formación, elaborando planes de formación en base a las necesidades reales detectadas en los Centros Gestores, impartiendo directamente una formación de índole general y descentralizando la formación sectorial.

Tercera: La formación como factor estratégico del cambio

La formación debe concebirse como un factor esencial y estratégico del cambio, potenciando la participación de los Centros Gestores en su diseño.

\section{Cuarta: Formación de directivos}

La formación debe favorecer la difusión de las nuevas tecnologías y la formación de directivos mediante la impartición de cursos de contenido técnico y gerencial en donde la formación teórica y práctica estén equilibradas.

\section{Quinta: La formación como elemento motivador}

Es necesario reforzar la incidencia de la formación en la carrera profesional, estableciendo una clara vinculación entre ambos aspectos, de suerte que por esta vía se potencie la motivación del funcionario.

Sexta: «Los cursillistas»

El actual sistema de cursos del INAP ha fomentado la aparición del «cursillista» que considera al INAP como una fábrica de «títulos» o «certificados», de los que únicamente se aprovecha el funcionario en detrimento de las necesidades de la organización. 
Séptima: El sistema de selección

Debe mejorarse el sistema de selección de los participantes en los cursos, debiendo primar a estos efectos las necesidades de la organización frente a los intereses del funcionario.

\section{Octava: Los formadores}

Debe potenciarse la «formación de los formadores», con la finalidad de tener un «cuadro» de profesores altamente cualificado que permita abordar con éxito la difusión de la «nueva cultura» administrativa y permita la «modernización» de la gestión.

Novena: Sistema de evaluación

Debe implantarse un sistema de evaluación de los cursos impartidos que tenga en cuenta las mejoras producidas en la organización a través del cambio cualitativo generado por la aplicación de las nuevas tecnologías impartidas a los gestores.

\section{Décima: Medios}

Deben mejorarse los medios para la formación aumentando las dotaciones presupuestarias, tanto sectoriales como globales.

\section{Undécima: Información}

Deben potenciarse las campañas de difusión e información tanto del plan general de formación como de los cursos sectoriales, como factor que coadyuve a la motivación del personal de la Administración pública. 